\title{
A strain gradient plasticity model of porous single crystal ductile fracture
}

\author{
Jean-Michel Scherer $^{\mathrm{a}, \mathrm{b}, *}$, Jacques Besson ${ }^{\mathrm{b}}$, Samuel Forest ${ }^{\mathrm{b}}$, Jérémy Hure ${ }^{\mathrm{a}}$, \\ Benoît Tanguy ${ }^{a}$ \\ ${ }^{a}$ Université Paris-Saclay, CEA, Service d'Étude des Matériaux Irradiés, 91191, Gif-sur-Yvette, France \\ b MINES ParisTech, PSL University, MAT - Centre des matériaux, CNRS UMR 7633, BP 8791003 Evry, France
}

\section{A R T I C L E I N F O}

\section{Keywords:}

Ductile failure

Crystal plasticity

Strain gradient plasticity

Fracture toughness anisotropy

\begin{abstract}
A B S T R A C T
A strain gradient void-driven ductile fracture model of single crystals is proposed and applied to simulate crack propagation in single and oligo-crystal specimens. The model is based on a thermodynamical framework for homogenized porous solids unifying and generalizing existing thermodynamical formulations. This porous single crystal ductile fracture model relies on a multi-surface representation of porous crystal plasticity in which the standard Schmid law is enhanced to account for porosity, including void growth and void coalescence mechanisms. A new criterion to detect the onset of void coalescence in porous single crystals is proposed and validated by comparison to porous single crystal unit-cell simulations. This criterion can either be used as an additional yield surface or it can be used to follow the well established GursonTvergaard-Needleman approach based on an effective porosity to model void coalescence. The strain gradient formulation relies on a Lagrange multiplier based relaxation of strain gradient plasticity. Material points simulations are performed in order to depict the elementary features of the porous single crystal ductile fracture model without strain gradient effects. The model is then applied to the simulation of plane strain single crystal specimen loaded in tension up to failure. The regularization ability and convergence with mesh refinement are demonstrated. Finally two- and three-dimensional simulations of ductile fracture of single and oligo-crystal specimens are presented. The significant influence of plastic anisotropy on the crack path, ductility and fracture toughness is highlighted.
\end{abstract}

\section{Introduction}

Modelling ductile fracture of metallic alloys is a major topic in the field of mechanical engineering. Multiple mechanisms can lead to ductile fracture (Noell et al., 2018) that is commonly characterized by significant local inelastic deformation prior to material separation and formation of free surfaces. One main mechanism is related to nucleation, growth and coalescence of voids in the bulk material. The seminal works of McClintock (1968), Rice and Tracey (1969), Green (1972), Gurson (1977), Rousselier (1981) and Thomason (1985) paved the way to the derivation of continuum mechanics models of ductile fracture provoked by combined plastic deformation and evolution of voids. The key ingredient of these models consists in introducing a damage scalar variable, representing the volume fraction of voids. The evolution of the damage variable is driven by the local loading state of the material. In order to efficiently reproduce failure after significant plastic deformation, these models rely on an elegant way of decreasing the load bearing capacity of the material when the amount of damage increases. This is accomplished by deriving yield potentials for

\footnotetext{
* Corresponding author at: Université Paris-Saclay, CEA, Service d'Étude des Matériaux Irradiés, 91191, Gif-sur-Yvette, France. E-mail address: jean-michel.scherer@mines-paristech.fr (J.-M. Scherer).
} 
which the elastic domain shrinks when damage increases. This approach encompasses two major requirements: (1) to define the appropriate evolution of the damage variable and (2) to get the appropriate dependency of the yield criteria upon damage. In the context of porous plasticity (see the reviews by Benzerga and Leblond, 2010; Besson, 2010; Pineau et al., 2016), increase of damage is mainly governed by void nucleation, growth and coalescence. Some authors also proposed extensions involving contribution of void shearing in the effective damage variable evolution (Nahshon and Hutchinson, 2008), although in that way, in general, the link of the damage variable to the void volume fraction is lost. Deriving effective yield criteria of porous solids was achieved by using mainly three techniques. The first, conducted by Gurson (1977), involves limit analysis of an idealized porous unit-cell. The second, proposed by Rousselier (1981, 1987, 2001), calls upon thermodynamical considerations (Germain et al., 1983). The third, followed for example by Danas and Castañeda (2009) is based on variational homogenization methods. Early models were extensively enriched to improve their accuracy for instance by introducing fitting parameters (Tvergaard, 1981, 1982; Tvergaard and Needleman, 1984). Extensions were also developed to account for shape (Gologanu et al., 1995), orientation (Cao et al., 2015) and size of voids (Dormieux and Kondo, 2010; Gallican and Hure, 2017). In the homogenization procedure, the behaviour of the so-called matrix material that surrounds the voids plays a paramount role. Gurson originally considered an isotropic rigid perfectly plastic matrix material. Gurson's approach was later generalized to take isotropic hardening and kinematic hardening into account (Mear and Hutchinson, 1985; Besson and Guillemer-Neel, 2003; Morin et al., 2017). Other studies focused on deriving effective yield criteria of porous materials with a plastic anisotropic matrix material (Benzerga and Besson, 2001; Morin et al., 2015; Keralavarma and Chockalingam, 2016). Recent studies also investigate the role of size-effects in porous metals (Monchiet and Kondo, 2013; Holte et al., 2019; Niordson and Tvergaard, 2019; Scherer et al., 2019).

In most metallic alloys voids nucleate at inclusions or precipitates by debonding or cracking (Babout et al., 2004). These defects can be within the bulk of grains. In this case, voids are individually surrounded by single crystals at short or even intermediate distances. Recent model experiments were carried out on polycrystal stainless steel tensile specimens containing holes drilled by focused ion beam (FIB) inside grains (Barrioz et al., 2019). These experiments confirmed the importance of crystal orientation on the plastic behaviour of the material surrounding the voids. Although the anisotropic nature of plasticity in single crystals could be captured to some extent by anisotropic yield criteria (Hill, 1948; Nouailhas and Cailletaud, 1992; Gambin, 1992), such approximations are known to fail for some complex loading paths. Furthermore, single crystal porous unit-cell simulations (Potirniche et al., 2006; Ha and Kim, 2010; Yerra et al., 2010; Han et al., 2013; Ling et al., 2016; Selvarajou et al., 2019) have shown the strong effect of crystal plasticity anisotropy on void growth and coalescence. Nevertheless, since the early work by Mori and Meshii (1969), only a few studies were devoted to develop models for porous single crystals able to describe the damage process up to failure. Single crystal void growth models were settled by Crépin et al. (1996), Han et al. (2013), Mbiakop et al. (2015), Ling et al. (2016), Song and Castañeda (2017) and Paux et al. (2018). Even fewer studies deal with void coalescence in single crystals (Yerra et al., 2010; Hure, 2019). A comprehensive model combining void growth and void coalescence criteria in porous single crystals is still lacking. Such a model would permit to assess the role of crystal plasticity anisotropy on ductility and toughness at the scale of the microstructure of metallic alloys.

Most ductile fracture models predict a softening regime at incipient final failure. Softening occurs in these models on account of damage variable increase which in turn reduces the size of the elastic domain. As a result softening induces localization and localization promotes damage acceleration. Although such a behaviour might be in agreement with underlying physical mechanisms it also entails the major issue of causing ill-posedness of the boundary value problem as reported by Bažant et al. (1984) and Lorentz and Benallal (2005). From a numerical point of view, solving the governing equations, for example by finite elements, results in the absence of convergence of the results when the mesh size is decreased. Several approaches were followed to bypass or overcome this issue. In Xue et al. (2010) and Achouri et al. (2013) mesh size is treated as a material parameter used to control the characteristic length of post-localization regime. Another technique used to introduce a material length scale consists in using the intrinsically size-dependent phase field method as in Miehe et al. (2016). Alternatively, theories developed in the context of non-local continua were also successfully applied in order to regularize localization predicted in ductile fracture simulations. Non-local theories, based either on integral or gradient formulations, naturally incorporate one or several length scales. In the context of ductile fracture, these lengths can be used to drive the evolution of the size of the damaged area in the post-localization regime. Size-dependent modelling of ductile fracture not only amounts to the choice of a non-local theory, but also to the choice of one or several appropriate nonlocal variables. Some authors used the damage variable to carry non-local effects (Tvergaard and Needleman, 1995; Ramaswamy and Aravas, 1998; Håkansson et al., 2006). Alternatively others used as non-local variables strain quantities such as the volumetric equivalent plastic strain in Zybell et al. (2014) and Nguyen et al. (2020), the equivalent plastic strain in Payet et al. (2012), Lorentz et al. (2008) and Nguyen et al. (2020), the strain tensor in Enakoutsa and Leblond (2009) or the matrix equivalent plastic strain in Nguyen et al. (2020). It is common that several non-local variables are used. Despite the more important numerical effort it requires, it is mostly necessary in order to be able to regularize localization for all types of loading paths (Nguyen et al., 2016, 2020).

The thermodynamics of continuum damage mechanics is extensively studied in the domain of geophysics and civil engineering, where rocks and soils contain defects (pores, cracks, etc.) which may or not be filled with fluids influencing their mechanical behaviour (Chaboche, 1988; Coussy, 2004; Kachanov, 2013). However literature covering thermodynamics of porous metallic alloys remains very scarce. Yet in his seminal work Rousselier (1981) was able to design a mechanical model of ductile failure based on very simple thermodynamical considerations. Furthermore similarities between this model and models derived with different approaches are remarkable. A few other thermodynamical settings were developed in Enakoutsa et al. (2007), Besson (2009), Bouby and Kondo (2017) and Pascon and Waisman (2020). Yet, these models rely on assumptions and have limitations discussed in Scherer (2020), in which a unifying framework is also proposed. 
The first and foremost challenge tackled in the present work is to address the formulation, implementation and application of a newly developed ductile fracture model for porous crystals. The main novel achievements of this work are numerical simulations performed with this model to assess the significant role of crystal plasticity anisotropy on ductility and toughness at the scale of the microstructure. The proposed model is implemented in a finite strain framework on the basis of the void growth model developed in Han et al. (2013) and Ling et al. (2016) and also used and extended by Khadyko et al. (2021) and Frodal et al. (2021). Then, an original coalescence criterion, adapted for intervoid necking in single crystals, is proposed and validated. In keeping with the multi-mechanism plasticity framework proposed by Besson (2009), void growth yield criteria will be combined to a void coalescence criterion to obtain a so-called multi-surface model. This method is compared to the well established Gurson-TvergaardNeedleman (Tvergaard and Needleman, 1984) approach for void growth and coalescence which relies on an effective porosity. The present work takes advantage of the strain gradient crystal plasticity model developed (without damage) and compared to the micromorphic approach in Scherer et al. (2020). This finite strain formulation of strain gradient plasticity is based on a Lagrange multiplier method already successfully applied by Zhang et al. (2018) for isotropic materials in the context of ductile fracture. For numerical efficiency a single scalar non-local variable is used, although from a theoretical point of view a tensorial non-local variable could be considered. A sound thermodynamical framework for porous plasticity which unifies existing theories was derived in Scherer (2020). This framework works as a prerequisite in order to be able to introduce strain gradient effects and couplings in the constitutive equations of the proposed porous crystal plasticity model.

The outline is as follows. In Section 2 a thermodynamical framework designed for multi-mechanism strain gradient porous plasticity is presented. The chosen gradient enhanced principle of virtual power, free energy potential and dissipation potentials are exposed in order to derive an original comprehensive model of ductile fracture in single crystals. Void growth and the newly proposed void coalescence flow potentials are described in Section 3 with their associated evolution equations. In Section 4 the single crystal material behaviour is detailed and the void coalescence onset criterion is validated. Two different approaches to account for void coalescence are then discussed on the basis of material points simulations. The ability of the proposed model to regularize ductile fracture is then demonstrated. In Section 5 2D and 3D ductile fracture simulations of single and oligo-crystals structures are presented. The main outcomes and prospects are listed in Section 6.

\section{Multi-mechanism based strain gradient porous plasticity framework}

A multi-mechanism deformation framework for homogenized porous materials established at finite strain is presented in the context of growth and coalescence of voids in materials with non-local gradient effects. Note that this framework is well suited for, but not restricted to crystal plasticity and can thus be used for other applications in which multiple plastic deformation mechanisms are involved.

\subsection{Void growth and void coalescence in single crystals}

A multiplicative decomposition of the deformation gradient $\underset{\sim}{\boldsymbol{F}}=\partial \underline{\boldsymbol{x}} / \partial \underline{\boldsymbol{X}}$ in an elastic part $\underset{\sim}{\boldsymbol{E}}$ and a plastic part $\underset{\sim}{\boldsymbol{P}}$ is assumed: $\underset{\sim}{\boldsymbol{F}}=\underset{\sim}{\boldsymbol{E}} . \underset{\sim}{\boldsymbol{P}}$. The elastic deformation rate $\underset{\sim}{\boldsymbol{L}}=\underset{\sim}{\boldsymbol{E}} \cdot \underset{\sim}{\boldsymbol{E}}{ }^{-1}$ and plastic deformation rate $\underset{\sim}{\boldsymbol{L}}{ }^{p}=\underset{\sim}{\boldsymbol{P}} \cdot \underset{\sim}{\boldsymbol{P}}{ }^{-1}$ are introduced such that $\underset{\sim}{\boldsymbol{L}}=\underset{\sim}{\boldsymbol{\boldsymbol { F }}} \cdot \underset{\sim}{\boldsymbol{F}} \boldsymbol{\sim}^{-1}=$ $\tilde{\sim}^{\boldsymbol{\tau}}+\underset{\sim}{\boldsymbol{E}} \cdot \underbrace{\tilde{L}}_{\sim} \cdot{ }_{\sim}^{\boldsymbol{E}} \boldsymbol{\sim}^{-1}$. Following the work developed by Ling et al. (2016), a void growth deformation mechanism is considered for each slip system of the crystal. An additional deformation mechanism is introduced to account for void coalescence. Coalescence is a phenomenon which mostly involves activation of many slip systems inside the ligaments separating coalescing voids (Barrioz et al., 2019). Coalescence is therefore not decomposed in a sum of contributions of individual slip systems. Therefore, for a crystal having $N$ slip systems, the inelastic deformation rate is

$$
\underset{\sim}{\dot{\boldsymbol{P}}} \cdot{\underset{\sim}{\boldsymbol{P}}}^{-1}=\sum_{s=1}^{N} \underset{\sim s}{\boldsymbol{L}_{s}^{p}}+\underset{\sim c}{\boldsymbol{L}}
$$

where the terms in the sum account for plastic slip and void growth on each slip system, and $\underset{\sim}{\mathbf{L}}$ is the inelastic rate associated to void coalescence. For each deformation mechanism a scalar deformation rate can be introduced. They will be denoted $\dot{\gamma}^{s}$ for void growth and $\dot{\gamma}^{c}$ for void coalescence. Note that $\dot{\gamma}^{c}$ is not to be interpreted as a slip rate, but more as an equivalent plastic strain rate, since void coalescence is not a mechanism decomposed on slip systems. An accumulated inelastic deformation variable is introduced as

$$
\gamma_{\text {cum }}=\int_{0}^{t}\left(\sum_{s=1}^{N}\left|\dot{\gamma}^{s}\right|+\left|\dot{\gamma}^{c}\right|\right) \mathrm{d} t
$$

The flow rules for void growth and coalescence are presented in Section 3.

\subsection{Gradient enhanced principle of virtual power}

In the spirit of the model developed by Wulfinghoff and Böhlke (2012) it is assumed that the gradient effects operate on the accumulated plasticity scalar variable $\gamma_{\text {cum }}$. Following Fleck and Hutchinson (1997), Forest and Sievert (2003) and Gurtin and Anand (2009), upon neglecting the contribution of body forces, for any material subset $D_{0}$ and its boundary $\partial D_{0}$, in the reference configuration, an enriched principle of virtual power is stated

$$
\int_{D_{0}}\left(\underset{\sim}{\boldsymbol{S}}: \underset{\sim}{\dot{\boldsymbol{F}}}+S \dot{\gamma}_{\gamma_{c u m}}+\underline{\boldsymbol{M}} \cdot \underline{\dot{\boldsymbol{K}}}\right) \mathrm{d} V_{0}=\int_{\partial D_{0}}\left(\underline{\boldsymbol{T}} \cdot \underline{\dot{\boldsymbol{u}}}+M \dot{\gamma}_{c u m}\right) \mathrm{d} S_{0} \quad \forall \underline{\dot{u}}, \forall \dot{\gamma}_{c u m}, \forall D_{0}
$$


where $\mathrm{d} S_{0}$ and $\mathrm{d} V_{0}$ represent infinitesimal surface and volume elements respectively. $\underset{\sim}{S}$ is the first Piola-Kirchhoff (Boussinesq) stress tensor related to the Cauchy stress tensor, $\underset{\sim}{\sigma}$, by $\underset{\sim}{\boldsymbol{S}}=\left(\rho_{0} / \rho\right) \underset{\sim}{\boldsymbol{\sigma}} \underset{\sim}{\boldsymbol{T}}$. Higher order stress scalar $\boldsymbol{S}$ and vector $\boldsymbol{M}$ are work conjugate to $\gamma_{\text {cum }}$ and $\underline{\boldsymbol{K}}=\operatorname{Grad} \gamma_{\text {cum }}$. The power of internal forces on the left-hand side of Eq. (3) is in equilibrium with the power of contact forces on the right-hand side. The traction vector is $\underline{T}$ and a higher order traction scalar $M$ is dual to $\gamma_{\text {cum }}$. From Eq. (3) one can develop the following balance equations and boundary conditions

$$
\begin{array}{rllll}
\operatorname{Div} \underline{\sim}=\underline{\boldsymbol{0}} & \forall \underline{X} \in D_{0} & \text { and } & \underline{\boldsymbol{T}}=\underline{\sim}_{\sim} \cdot \underline{\boldsymbol{n}}_{0} & \forall \underline{\boldsymbol{X}} \in \partial D_{0} \\
\operatorname{Div} \underline{\boldsymbol{M}}-S=0 & \forall \underline{\boldsymbol{X}} \in D_{0} & \text { and } & M=\underline{\boldsymbol{M}} \cdot \underline{\boldsymbol{n}}_{0} & \forall \underline{\boldsymbol{X}} \in \partial D_{0}
\end{array}
$$

where $\underline{\boldsymbol{n}}_{0}$ refers to the outward unit surface normal.

\subsection{Gradient enhanced free energy potential}

The first step to the definition of the material behaviour is the choice of a specific free energy density potential which depends on

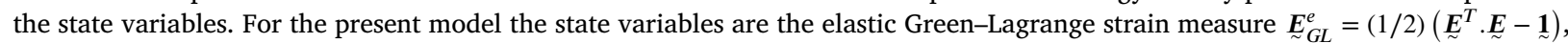
the accumulated plastic deformation $\gamma_{\text {cum }}$, its Lagrangian gradient $\underline{K}$, one hardening variable $r^{s}$ per slip system $s$ and the porosity $f$. The general form of the specific free energy potential can be written as follows

$$
\psi\left(\underset{\sim G L}{\boldsymbol{E}_{G L}^{e}}, \gamma_{\text {cum }}, \gamma_{\chi}, \underline{\boldsymbol{K}}, r^{s}, f\right)=\psi_{e}(\underset{\sim G L}{\boldsymbol{E}}, f)+\psi_{h f}\left(\gamma_{c u m}, r^{s}, f\right)+\psi_{g}\left(\gamma_{c u m}, \gamma_{\chi}, \underline{\boldsymbol{K}}, f\right)+\psi_{f}(f)
$$

where $\psi_{e}, \psi_{h f}, \psi_{g}$ and $\psi_{f}$ represent energy contributions of elasticity, strain hardening, strain gradient hardening and porosity respectively. For the sake of simplicity the dependence to $f$ is dropped in the following. For a detailed discussion on this topic the reader is referred to Enakoutsa et al. (2007), Besson (2009), Bouby and Kondo (2017), Pascon and Waisman (2020) and Scherer (2020). Quadratic potentials are used for the elastic and strain gradient contributions as follows

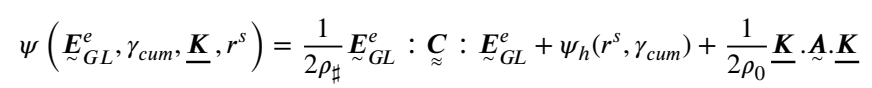

The scalar $\rho_{0}$ and $\left.\rho_{\sharp}=\rho_{0} / \operatorname{det} \underset{\sim}{\boldsymbol{P}}\right)$ respectively represent the volumetric mass density in the initial and intermediate configuration of the body. The intermediate configuration is defined as the configuration obtained after transformation by $\underset{\sim}{\boldsymbol{P}}$, while the current configuration is obtained after transformation by $\underset{\sim}{\boldsymbol{F}}$. Note that in the context of porous plasticity $\operatorname{det}(\underset{\sim}{\boldsymbol{P}}) \neq 1$ in $\operatorname{\sim }$ eneral. $\rho_{\sharp}$ is $\operatorname{directly}$ linked to the porosity by the relation $\rho_{\sharp} / \rho_{0}=1 / \operatorname{det}(\underset{\sim}{\boldsymbol{P}})=(1-f) /\left(1-f_{0}\right)$, with $f_{0}$ the initial porosity. Yet, since the dependence of $\psi$ with respect to $f$ is omitted, the volumetric mass density in the intermediate configuration $\rho_{\sharp}$ enters Eq. (7) as a parameter, but not as variable. The material parameter $\underset{\sim}{\boldsymbol{A}}$ is a second order tensor of higher order moduli. For a material with cubic symmetry $\underset{\sim}{\boldsymbol{A}}$ reduces to $A \mathbf{\sim}$, where $A$ is the single higher order modulus in this case. From a numerical perspective, the implementation of such a formulation is challenging. The behaviour of a material point is no longer independent from the value of the internal variables in its neighbourhood. The gradient of $\gamma_{\text {cum }}$ enters indeed the constitutive equations. In order to overcome this difficulty the variable $\gamma_{\text {cum }}$ can be duplicated in an auxiliary variable $\gamma_{\chi}$ treated as an additional degree of freedom (Zhang et al., 2018). Both variables have the same physical interpretation, but, in a finite element setting for instance, the former is defined at integration points while the latter is defined at nodes. In order to enforce weakly the equality of these variables, the free energy density is extended with two additional terms as

$$
\begin{aligned}
& \psi\left(\underset{\sim G L}{\boldsymbol{E}_{G L}^{e}}, \gamma_{\text {cum }}, \gamma_{\chi}, \underline{\boldsymbol{K}}_{\chi}, r^{s}, \lambda\right)=\frac{1}{2 \rho_{\sharp}} \underset{\sim G L}{\boldsymbol{E}}:^{e} \underset{\sim}{\boldsymbol{C}}: \underset{\sim G L}{\boldsymbol{E}_{G}^{e}}+\psi_{h}\left(r^{s}, \gamma_{\text {cum }}\right) \\
& +\frac{A}{2 \rho_{0}} \underline{K}_{\chi} \cdot \underline{K}_{\chi}+\frac{\lambda}{\rho_{0}}\left(\gamma_{\text {cum }}-\gamma_{\chi}\right)+\frac{\mu_{\chi}}{2 \rho_{0}}\left(\gamma_{\text {cum }}-\gamma_{\chi}\right)^{2}
\end{aligned}
$$

where $\lambda$ is a Lagrange multiplier which enforces $\gamma_{\chi}$ and $\gamma_{c u m}$ to be equal and $\mu_{\chi}$ is a Lagrangian penalization modulus enhancing coercivity of the model. The gradient of $\gamma_{\text {cum }}$ is now replaced by the gradient of the auxiliary variable $\underline{\boldsymbol{K}} \underline{\chi}_{\chi}=$ Grad $\gamma_{\chi}$. This formulation can be interpreted as a way of imposing an internal constraint on $\gamma_{\chi}$ (Bertram and Glüge, 2016). From the 1st and 2nd principle of thermodynamics the Clausius-Duhem inequality is written

$$
d=\frac{\underset{\sim}{\boldsymbol{S}}}{\rho_{0}}: \underset{\sim}{\dot{\boldsymbol{F}}}+\frac{S}{\rho_{0}} \dot{\gamma}_{\chi}+\frac{\underline{\boldsymbol{M}}}{\rho_{0}} \cdot \dot{\boldsymbol{K}}_{\chi}-\dot{\psi} \geq 0
$$

The first term of equation Eq. (9) can be decomposed into elastic and plastic contributions. The mechanical dissipation therefore becomes

$$
\begin{aligned}
& d=\left(\frac{\underset{\sim}{\boldsymbol{\Pi}^{e}}}{\rho_{\sharp}}-\frac{\partial \psi}{\partial{\underset{\sim}{\boldsymbol{E}}}_{G L}^{e}}\right):{\underset{\sim}{\dot{\boldsymbol{E}}_{G L}^{e}}}^{e}+\left(\frac{S}{\rho_{0}}-\frac{\partial \psi}{\partial \gamma_{\chi}}\right) \dot{\gamma}_{\chi}+\left(\frac{\underline{\boldsymbol{M}}}{\overline{\rho_{0}}}-\frac{\partial \psi}{\partial \underline{\boldsymbol{K}}_{\chi}}\right) \cdot \dot{\boldsymbol{K}}_{\chi} \\
& +\frac{\underset{\sim}{\boldsymbol{\Pi}}}{\rho_{\sharp}}:\left(\underset{\sim}{\dot{\boldsymbol{P}}} \underset{\sim}{\boldsymbol{P}^{-1}}\right)-\sum_{s=1}^{N} \frac{\partial \psi_{h}}{\partial r^{s}} \dot{\boldsymbol{r}}^{s}-\frac{\partial \psi}{\partial \gamma_{\text {cum }}} \dot{\gamma}_{\text {cum }}-\frac{\partial \psi}{\partial \lambda} \dot{\lambda} \geq 0
\end{aligned}
$$

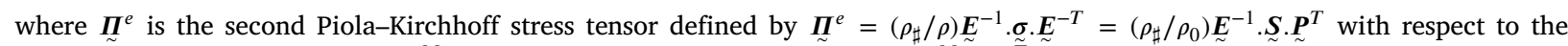

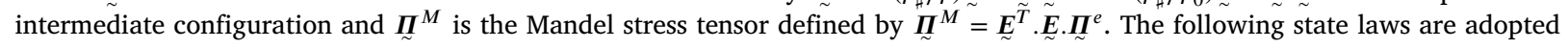

$$
\underset{\sim}{\boldsymbol{\Pi}}{ }^{e}=\rho_{\sharp} \frac{\partial \psi}{\partial \underset{\sim G L}{\boldsymbol{E}_{G L}^{e}}}=\underset{\sim}{\boldsymbol{C}}: \underset{\sim G L}{\boldsymbol{E}}
$$




$$
\begin{aligned}
S & =\rho_{0} \frac{\partial \psi}{\partial \gamma_{\chi}}=\lambda-\mu_{\chi}\left(\gamma_{\text {cum }}-\gamma_{\chi}\right)=\Delta_{\chi}-\mu_{\chi} \gamma_{\text {cum }} \\
\underline{\boldsymbol{M}} & =\rho_{0} \frac{\partial \psi}{\partial \underline{\boldsymbol{K}}_{\chi}}=A \underline{\boldsymbol{K}}_{\chi}
\end{aligned}
$$

For convenience the scalar stress $\Delta_{\chi}=\lambda+\mu_{\chi} \gamma_{\chi}$ is introduced. By definition $\partial \psi / \partial \lambda$ must vanish when the constraint $\gamma_{\text {cum }}=\gamma_{\chi}$ is met therefore

$$
\frac{\partial \psi}{\partial \lambda} \dot{\lambda}=\left(\gamma_{\text {cum }}-\gamma_{\chi}\right) \frac{\dot{\lambda}}{\rho_{0}}=0
$$

and the residual mechanical dissipation follows

$$
d=\frac{\underset{\sim}{\boldsymbol{\Gamma}}}{\rho_{\sharp}}:\left(\underset{\sim}{\underset{\sim}{\boldsymbol{P}}} \boldsymbol{\sim}^{-1}\right)-\sum_{s=1}^{N} \frac{\partial \psi_{h}}{\partial r^{s}} \dot{r}^{s}-\left(\frac{\mu_{\chi} \gamma_{c u m}-\Delta_{\chi}}{\rho_{0}}+\frac{\partial \psi_{h}}{\partial \gamma_{c u m}}\right) \dot{\gamma}_{c u m}
$$

Here it is postulated that rates of hardening variables are proportional to slip rates on each slip system, i.e. $\dot{r}^{s}=g_{s}\left(r^{s}\right)\left|\dot{\gamma}^{s}\right|$. The thermodynamic forces are defined as

$$
\begin{aligned}
\frac{R_{s}}{\rho_{\sharp}} & =\frac{\partial \psi_{h}}{\partial r^{s}} g_{s}\left(r^{s}\right) \\
\frac{R_{\text {cum }}}{\rho_{\sharp}} & =\frac{\partial \psi_{h}}{\partial \gamma_{\text {cum }}}
\end{aligned}
$$

The strain gradient term $\Delta_{\chi}$ plays therefore the role of an isotropic hardening or softening contribution to the mechanical dissipation.

\subsection{Gradient enhanced dissipation potentials}

In the present framework the dissipation potential is a function of thermodynamical forces and the state variables can intervene as parameters. For the void growth and void coalescence mechanisms the individual potentials for the mechanisms $i=\llbracket 1, N+1 \rrbracket$ are

$$
\Omega_{i}=\Omega_{i}\left(\frac{\underset{\sim}{\boldsymbol{I}^{M}}}{\rho_{\sharp}}, \frac{R_{i}}{\rho_{\sharp}}, \frac{R_{\text {cum }}}{\rho_{\sharp}} ; \underset{\sim G L}{\boldsymbol{E}_{G L}}, r_{i}, \gamma_{\text {cum }}, \gamma_{\chi}, \lambda\right)=\Lambda\left(\phi^{i}\right)
$$

where $\Lambda$ is the viscosity function and $\phi^{i}$ is the flow potential associated to mechanism $i$. Flow potentials for void growth and void coalescence in porous (visco-)plastic single crystals are described in following section.

\section{Porous crystal (visco-)plasticity}

\subsection{Void growth}

For void growth mechanisms in single crystals, an extension of the implicit definition of effective resolved shear stresses $\tau_{*}^{s}$ established at small strains for porous single crystals by Han et al. (2013) and extended to finite strains by Ling et al. (2016) is adopted

$$
\tau_{*}^{s} \text { such that } \phi^{s}=\left(\frac{\tau^{s}}{\tau_{*}^{s}}\right)^{2}+\alpha \frac{2}{45} f\left(\frac{\Pi_{e q}^{M}}{\tau_{*}^{s}}\right)^{2}+2 q_{1} f \cosh \left(q_{2} \sqrt{\frac{3}{20}} \frac{\Pi_{m}^{M}}{\tau_{*}^{s}}\right)-1-\left(q_{1} f\right)^{2} \stackrel{\text { def }}{\equiv} 0
$$

where ${\underset{\sim}{\boldsymbol{\Pi}}}^{M}={\underset{\sim}{\boldsymbol{\Gamma}}}^{M^{\prime}}+\Pi_{m}^{M} \underset{\sim}{\mathbf{1}}$, with ${\underset{\sim}{\boldsymbol{\Pi}}}^{M^{\prime}}$ and $\Pi_{m}^{M} \underset{\sim}{\mathbf{1}}$ the deviatoric and hydrostatic part of ${\underset{\sim}{\boldsymbol{\Pi}}}^{M}$ respectively, and $\tau^{s}=\underline{\boldsymbol{\Pi}}^{M}:\left(\underline{\boldsymbol{m}}^{s} \otimes \underline{\boldsymbol{n}}^{s}\right)$ with $\underline{\boldsymbol{m}}^{\tilde{s}}$ and $\underline{\boldsymbol{n}}^{s}$ the gliding direction and normal to slip plane for system $s$ respectively (with $\underline{\boldsymbol{m}}^{s} \perp \underline{\boldsymbol{n}}^{s}$ ). $\Pi_{e q}^{M}$ is defined as the von Mises norm of $\underset{\sim}{\boldsymbol{\Pi}}{ }^{M}$, i.e. $\Pi_{e q}^{M}=\sqrt{(3 / 2) \boldsymbol{\sim}^{M^{\prime}}: \boldsymbol{\sim}^{M^{\prime}}}$. With this definition, the effective stresses $\tau_{*}^{s}$ are positive. The flow potentials are then chosen as

$$
\tilde{\phi}^{s}=(1-f)\left(\tau_{*}^{s}-R_{s}-R_{\text {cum }}-\frac{\rho_{\sharp}}{\rho_{0}}\left(\mu_{\chi} \gamma_{\text {cum }}-\Delta_{\chi}\right)-\tau_{0}^{s}\right)
$$

where $\tau_{0}^{s}$ is the initial critical resolved shear stress of system $s$. The terms $R_{s}$ and $R_{\text {cum }}$ correspond to conventional hardening contributions. $R_{s}$ can for example be used to model dislocations based hardening. $R_{\text {cum }}$ can for instance be used to introduce an additional phenomenological hardening. The term $\rho_{\sharp} / \rho_{0}\left(\mu_{\chi} \gamma_{c u m}-\Delta_{\chi}\right)$ corresponds to the strain gradient contribution. Note that, in general, it cannot be excluded that, for large strain gradient effects, the effective flow stresses, defined by $\tau_{0}^{s}+R_{s}+R_{\text {cum }}+$ $\left(\rho_{\sharp} / \rho_{0}\right)\left(\mu_{\chi} \gamma_{\text {cum }}-\Delta_{\chi}\right)$, become negative (Ling et al., 2018). In practice, the positive part of the effective flow stress is used in the flow potentials. The evolution laws for void growth follows

$$
\underset{\sim}{\boldsymbol{L}_{s}^{p}}=-\frac{\mathrm{d} \Lambda}{\mathrm{d} \tilde{\phi}^{s}} \frac{\partial \tilde{\phi}^{s}}{\partial\left(-\frac{\boldsymbol{\Pi}^{M}}{\rho_{\sharp}}\right)}=(1-f) \dot{\gamma}^{s}{\underset{\sim}{*}}_{*}^{s}
$$




$$
\dot{r}_{s}=-\frac{\mathrm{d} \Lambda}{\mathrm{d} \tilde{\phi}^{s}} \frac{\partial \tilde{\phi}^{s}}{\partial\left(\frac{R_{s}}{\rho \sharp}\right)}=-\dot{\gamma}^{s} \frac{\partial \tilde{\phi}^{s}}{\partial\left(\frac{R_{s}}{\rho \sharp}\right)}
$$

with $d \Lambda / d \tilde{\phi}^{s}=\dot{\gamma}^{s}$ and where the normal tensor ${\underset{\sim}{*}}_{*}^{s}$ already derived in Ling et al. (2016) is introduced as

$$
\underset{\sim}{\boldsymbol{N}_{*}^{s}}=\frac{\partial \tau_{*}^{s}}{\partial\left(-\frac{\boldsymbol{\Pi}^{M}}{\rho_{\sharp}}\right)}=-\left(\frac{\partial \phi^{s}}{\partial \tau_{*}^{s}}\right)^{-1} \frac{\partial \phi^{s}}{\partial\left(\frac{\boldsymbol{\sim}^{M}}{\rho_{\sharp}}\right)}
$$

with

$$
\frac{\partial \phi^{s}}{\partial \tau_{*}^{s}}=-2 \frac{\tau^{s 2}}{\tau_{*}^{s 3}}-\frac{4}{45} \alpha f \frac{\Pi_{e q}^{M^{2}}}{\tau_{*}^{s 3}}-2 \sqrt{\frac{3}{20}} q_{1} q_{2} f \frac{\Pi_{m}^{M}}{\tau_{*}^{s 2}} \sinh \left(q_{2} \sqrt{\frac{3}{20}} \frac{\Pi_{m}^{M}}{\tau_{*}^{s}}\right)
$$

and

$$
\frac{\partial \phi^{s}}{\partial\left(\underline{\sim}_{\rho_{\sharp}}^{\boldsymbol{\Pi}}\right)}=2 \frac{\tau^{s}}{\tau_{*}^{s 2}}\left(\underline{\boldsymbol{m}}^{s} \otimes \underline{\boldsymbol{n}}^{s}\right)+\frac{2}{15} \alpha f \frac{1}{\tau_{*}^{s 2}}{\underset{\sim}{\boldsymbol{\Pi}}}^{M^{\prime}}+\frac{2}{3} \sqrt{\frac{3}{20}} \frac{q_{1} q_{2} f}{\tau_{*}^{s}} \sinh \left(q_{2} \sqrt{\frac{3}{20}} \frac{\Pi_{m}^{M}}{\tau_{*}^{s}}\right) \underset{\sim}{\mathbf{1}}
$$

\subsection{Void coalescence}

A criterion to detect onset of coalescence by intervoid necking in single crystals was proposed by Yerra et al. (2010). This criterion is based on the well known criterion by Thomason (1985) which can be expressed with the function

$$
\phi^{c}=\sigma_{I}-C_{f} \sigma_{*}^{g}
$$

where $\sigma_{I}$ is the stress orthogonal to the coalescence plane defined by its normal vector $\underline{e}_{I}$, while $\sigma_{*}^{g}$ represents the effective flow stress of the matrix during void growth which will be discussed in the following. $C_{f}$ is a concentration factor. In general, the plane in which coalescence takes place is not known a priori. Therefore it is usually necessary to test the criterion over a wide range of directions in order to determine the plane in which coalescence will initiate at first. To alleviate this difficulty, it is assumed that the normal to the coalescence plane coincides with the direction of the largest eigenvalue of the symmetric Cauchy stress tensor $\sigma$. The scalar $\sigma_{I}$ is therefore interpreted as the maximum principal Cauchy stress and $\underline{e}_{I}$ is the associated eigenvector. This assumption was also proposed by Nguyen et al. (2020). The principal stress $\sigma_{I}$ satisfies $\sigma_{I}=\bar{\sigma}_{\sim}:\left(\underline{\boldsymbol{e}}_{I} \otimes \underline{\boldsymbol{e}}_{I}\right)$, with $\underset{\sim}{\boldsymbol{\sigma}}=(1 / \operatorname{det}(\underset{\sim}{\boldsymbol{E}})) \underset{\sim}{\boldsymbol{E}}{ }^{-T} . \underset{\sim}{\boldsymbol{\Pi}}{ }^{M} . \underset{\sim}{\boldsymbol{E}}{ }^{T}$. As for the void growth model presented above, the derivation of a coalescence criterion as in Eq. (26) relies on the analysis of the behaviour of a porous unit-cell. In the following we consider an initially orthorhombic unit-cell containing a centered spherical void. Due to the applied deformation, the cell and void shapes evolve and that affects the coefficient $C_{f}$ which is a function of cell and void geometries. To characterize these geometries, the cell aspect ratio $\lambda_{c}$, void aspect ratio $W$ and normalized intervoid ligament size $\chi$ are often introduced. For a spheroidal void of semi-axes $a_{1}$ and $a_{2}$ in a tetragonal unit-cell of dimensions $L_{1}$ and $L_{2}$ they are respectively expressed as

$$
\lambda_{c}=\frac{L_{1}}{L_{2}} \quad W=\frac{a_{1}}{a_{2}} \quad \chi=\frac{2 a_{2}}{L_{2}}
$$

The porosity can be expressed in terms of these geometrical quantities

$$
f=\frac{\frac{4}{3} \pi a_{1} a_{2}^{2}}{L_{1} L_{2}^{2}}=\frac{\pi}{6} \frac{W \chi^{3}}{\lambda_{c}}
$$

Since $C_{f}$ depends on these quantities, equations characterizing their evolution with loading are needed. Deriving an accurate evolution equation for the void aspect ratio $W$ in porous single crystals is out of the scope of the present study. A first proposal based on variational limit analysis was provided by Mbiakop et al. (2015). However, for the sake of simplicity, the void aspect ratio $W$ will be fixed to 1 , which corresponds to voids remaining spherical. For an arbitrary small porous unit-cell, the cell aspect ratio $\lambda_{c}$ can be expressed with respect to its initial value $\lambda_{c}^{0}$, initial porosity $f_{0}$ and the cell normalized elongation $L_{1} / L_{1}^{0}$ which depends upon the deformation gradient tensor $\underset{\sim}{\boldsymbol{F}}$ as follows

$$
\begin{aligned}
& \stackrel{\sim}{\boldsymbol{F}}^{-1} \cdot \underline{\boldsymbol{L}}_{1}=\underline{\boldsymbol{L}}_{1}^{0}
\end{aligned}
$$

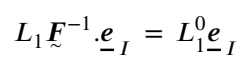

$$
\begin{aligned}
& \frac{L_{1}}{L_{1}^{0}}=\frac{1}{\sqrt{\left({\underset{\sim}{\boldsymbol{F}}}^{-1} \cdot \underline{\boldsymbol{e}}_{I}\right) \cdot\left(\underline{\sim}^{-1} \cdot \underline{\boldsymbol{e}}_{I}\right)}} \\
& \lambda_{c}=\lambda_{c}^{0}\left(\frac{L_{1}}{L_{1}^{0}}\right)^{\frac{3}{2}} \sqrt{\frac{1-f}{1-f_{0}}}
\end{aligned}
$$


To derive this expression it was assumed that the principal direction of the homogenization unit-cell coincides with the direction of the maximum principal Cauchy stress oriented by the eigenvector $\underline{\boldsymbol{e}}_{I}$. It appears from Eq. (28) that, assuming $W=1$, applying equation (32) and having an evolution equation for $f$ allows to compute $\chi$ with

$$
\chi=\left(\frac{6}{\pi} \lambda_{c} f\right)^{\frac{1}{3}}
$$

A particular form of $C_{f}$ as a function of $W$ and $\chi$ will be chosen in Section 4.2.2.

In Eq. (26) $\sigma_{*}^{g}$ represents the effective flow stress of the matrix during void growth. In Yerra et al. (2010) the authors account for hardening of the matrix by determining an effective flow stress in the vicinity of the void in the coalescence plane. They propose to perform an auxiliary computation on a single Gauss point with an identical crystal orientation and under an equibiaxial straining loading state which is representative of the loading during coalescence in the coalescence plane. The effective flow stress is then derived as the equivalent stress when the actual equivalent plastic deformation is reached in the auxiliary computation. Such a method is an elegant way to introduce hardening in Thomason's coalescence criterion. Nevertheless, the computational cost of performing these auxiliary simulations in order to determine the effective flow stress of the crystal matrix can become significant. In principle, at each iteration of the constitutive integration such a simulation should be done. Therefore two new approaches are proposed and described as follows. The main ingredient of these methods is to consider that at initiation of intervoid necking many slip systems are activated in the intervoid ligament and that hence the flow stress can be determined from an isotropic criterion. The Thomason criterion is reformulated as

$$
\begin{aligned}
\sigma_{*}^{c} \text { such that } \phi^{c} & =\sigma_{I}-C_{f} \sigma_{*}^{c} \stackrel{\text { def }}{\equiv} 0 \\
\tilde{\phi}^{c} & =(1-f)\left(\sigma_{*}^{c}-R_{\text {cum }}-\frac{\rho_{\sharp}}{\rho_{0}}\left(\mu_{\chi} \gamma_{\text {cum }}-\Delta_{\chi}\right)-\sigma_{*}^{g}\right)
\end{aligned}
$$

Eq. (34) defines an equivalent coalescence stress $\sigma_{*}^{c}$, while Eq. (35) is the effective coalescence flow potential. These equations are the coalescence counterpart of Eqs. (19) and (20) defined previously for void growth. Since many slip systems are active, the effective flow stress $\sigma_{*}^{g}$ of the crystal matrix can hence be approximated by the effective flow stress of an isotropic matrix. The latter can for instance be defined implicitly by a GTN-like equation

$$
\sigma_{*}^{g} \text { such that } \phi^{g}=\left(\frac{\Sigma_{e q}}{\sigma_{*}^{g}}\right)^{2}+2 q_{1}^{c} f \cosh \left(q_{2}^{c} \frac{3}{2} \frac{\Sigma_{m}}{\sigma_{*}^{g}}\right)-1-\left(q_{1}^{c} f\right)^{2} \stackrel{\text { def }}{\equiv} 0
$$

where $q_{1}^{c}$ and $q_{2}^{c}$ are parameters to be calibrated. $\sigma_{*}^{g}$ represents the effective flow stress of the matrix during void growth, thus it must be updated while coalescence is not taking place. However, once coalescence sets on, $\sigma_{*}^{g}$ is kept constant or follows a hardening law that will be discussed in Section 4.3. Once more, it cannot be excluded that, for large strain gradient effects, the effective flow stress, defined by $\sigma_{*}^{g}+R_{\text {cum }}+\left(\rho_{\sharp} / \rho_{0}\right)\left(\mu_{\chi} \gamma_{\text {cum }}-\Delta_{\chi}\right)$, becomes negative. In practice, the positive part of the effective flow stress is therefore used in the flow potential.

\subsubsection{Void coalescence deformation mechanism}

In the spirit of previous section, void coalescence can be interpreted as an independent deformation mechanism having its own yield surface and flow rule. Therefore, the coalescence criterion Eq. (35) can be regarded as a flow potential and it follows the evolution laws for void coalescence

$$
\underset{\sim c}{\boldsymbol{L}}=-\frac{\mathrm{d} \Lambda}{\mathrm{d} \tilde{\phi}^{c}} \frac{\partial \tilde{\phi}^{c}}{\partial\left(-\frac{\boldsymbol{\Pi}^{M}}{\rho_{\sharp}}\right)}=(1-f) \dot{\gamma}^{c} \underset{\sim}{\boldsymbol{N}^{c}}
$$

with $d \Lambda / d \tilde{\phi}^{c}=\dot{\gamma}^{c}$ and where the normal $\underset{\sim *}{N_{*}^{c}}$ is introduced such that

$$
\underset{\sim}{\boldsymbol{N}_{*}^{c}}=\frac{\partial \sigma_{*}^{c}}{\partial\left(-\frac{\boldsymbol{\Pi}_{\sim}^{M}}{\rho_{\sharp}}\right)}=-\left(\frac{\partial \phi^{c}}{\partial \sigma_{*}^{c}}\right)^{-1} \frac{\partial \phi^{c}}{\partial\left(\frac{\boldsymbol{\sim}^{M}}{\rho_{\sharp}}\right)}
$$

with

$$
\frac{\partial \phi^{c}}{\partial \sigma_{*}^{c}}=-C_{f}
$$

and

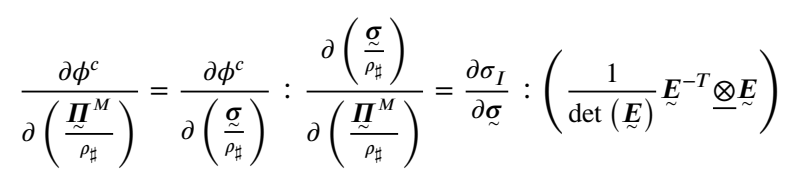

$$
\begin{aligned}
& =\left(\underline{\boldsymbol{e}}_{I} \otimes \underline{\boldsymbol{e}}_{I}\right):\left(\frac{1}{\operatorname{det}(\underset{\sim}{\boldsymbol{E}})}{\underset{\sim}{\boldsymbol{E}}}^{-T} \underline{\otimes} \underset{\sim}{\boldsymbol{E}}\right)
\end{aligned}
$$

according to the derivation rules given in Bertram (2012). 
The overall macroscopic plastic dissipation finally becomes

$$
\begin{aligned}
& \underset{\sim}{\boldsymbol{\Pi}}{ }^{M}:\left(\underset{\sim}{\dot{\boldsymbol{P}}} \underset{\sim}{\boldsymbol{P}^{-1}}\right)=(1-f) \sum_{s=1}^{N} \dot{\gamma}^{s} \boldsymbol{\sim}^{M}:{\underset{\sim}{\boldsymbol{N}}}_{*}^{s}+(1-f) \dot{\gamma}^{c}{\underset{\sim}{\boldsymbol{\prod}}}^{M}:{\underset{\sim}{\boldsymbol{N}}}_{*}^{c} \\
& =(1-f) \sum_{s=1}^{N} \dot{\gamma}^{s} \tau_{*}^{s}+(1-f) \dot{\gamma}^{c} \sigma_{*}^{c}
\end{aligned}
$$

This equation states the equivalence between macroscopically and microscopically dissipated energies. Combining Eqs. (15) and (42), the mechanical dissipation can eventually be formulated as a sum over all deformation mechanisms

$$
\begin{aligned}
d & =\frac{1-f}{\rho_{\sharp}} \sum_{s=1}^{N}\left(\tau_{*}^{s}-\frac{\rho_{\sharp}}{\rho_{0}}\left(\mu_{\chi} \gamma_{\text {cum }}-\Delta_{\chi}\right)-R_{s}-R_{\text {cum }}\right)\left|\dot{\gamma}^{s}\right| \\
& +\frac{1-f}{\rho_{\sharp}}\left(\sigma_{*}^{c}-\frac{\rho_{\sharp}}{\rho_{0}}\left(\mu_{\chi} \gamma_{\text {cum }}-\Delta_{\chi}\right)-R_{\text {cum }}\right)\left|\dot{\gamma}^{c}\right| \\
& =\frac{1}{\rho_{\sharp}} \sum_{s=1}^{N}\left(\tilde{\phi}^{s}+(1-f) \tau_{0}\right)\left|\dot{\gamma}^{s}\right|+\frac{1}{\rho_{\sharp}}\left(\tilde{\phi}^{c}+(1-f) \sigma_{*}^{g}\right)\left|\dot{\gamma}^{c}\right| \geq 0
\end{aligned}
$$

According to Eq. (44), positivity of the flow potentials $\tilde{\phi}^{k}$ associated to non-zero flow rates $\dot{\gamma}^{k}$ (with $k \in\{s, c\}$ ) is a sufficient condition to ensure positivity of the dissipation. The viscoplastic flow rules chosen in the following (see Eq. (50) for instance) satisfy this condition and thus guaranty the positivity of $d$.

\subsection{2. $f^{*}$-type void coalescence}

Void coalescence was described in previous sections as an individual plastic mechanism having its own yield surface. This approach is relatively straightforward from a modelling point of view. However, from a numerical perspective the implementation effort and computational costs associated to this approach can be significant. Another common approach in the literature consists in modelling void coalescence without extending the set of yield criteria. The method proposed by Tvergaard and Needleman (1984) introduces an effective definition of the porosity $f^{*}$ once a critical porosity $f_{c}$ is reached

$$
f^{*}= \begin{cases}f & \text { for } f \leq f_{c} \\ f_{c}+\frac{f_{u}^{*}-f_{c}}{f_{R}-f_{c}}\left(f-f_{c}\right) & \text { for } f>f_{c}\end{cases}
$$

where $f_{c}, f_{R}$ and $f_{u}^{*}$ are material parameters. $f_{c}$ describes the porosity at the onset of void coalescence. $f_{R}$ denotes the standard porosity at fracture, while $f_{u}^{*}$ is the effective porosity at fracture. With adequate numerical values of material parameters, Eq. (45) allows to artificially increase the effective porosity $f^{*}$ once coalescence sets on. As a consequence, the yield surfaces associated to void growth shrink at a greater rate leading to an accelerated stress drop. The advantage of utilizing Eq. (45) is that void coalescence can straightforwardly be incorporated in a numerical implementation of a void growth model. On the other hand, some drawbacks are the lack of physical foundation for the effective porosity and the necessity to know a priori the critical porosity at coalescence $f_{c}$. Furthermore, in such a formulation this parameter is assumed no to depend on the loading state.

In order to alleviate these two last drawbacks an hybrid formulation was proposed by Zhang et al. (2000). They proposed to revoke the ad hoc choice of $f_{c}$ and define it as the porosity reached when Thomason's coalescence onset criterion is met. In that manner, $f_{c}$ is continuously updated upon loading and becomes constant when void coalescence is reached. $f^{*}$-type void coalescence will rely on the coalescence criterion defined at Eq. (34) in order to obtain $f_{c}$ locally.

In the context of the strain gradient porous plasticity model presented in Section 2, assuming an effective porosity as in Eq. (45) does not involve major difficulties. The main point consists in replacing $f$ by $f^{*}$ in every equation, but one. The only equation in which $f$ is not replaced by $f^{*}$ is the evolution law of the porosity Eq. (48). However, it should be noted that since void coalescence is not treated as an independent plastic mechanism with its own yield surface, $\gamma^{c}$ is not defined anymore. As a consequence the accumulated plastic slip $\gamma_{\text {cum }}$ defined at Eq (2) and the auxiliary variable $\gamma_{\chi}$ do not account for void coalescence explicitly. However, since void growth criteria are affected by $f^{*}$, void coalescence still has an indirect effect on $\gamma_{\text {cum }}$ and $\gamma_{\chi}$.

It is well established in the literature that $f_{u}^{*}$ can be adjusted in order to obtain an adequate acceleration of the porosity when a $f^{*}$-type coalescence model is used (Zhang et al., 2000). In Fig. 2, the numerical value chosen for $f_{u}^{*}$, namely $67 \%$, leads to a relatively weak acceleration of porosity increase. Increasing $f_{u}^{*}$ would result in a sharper acceleration of porosity and thus in a faster drop of the stress. When coalescence is treated as an additional yielding mechanism it is less straightforward to control the slopes of porosity increase and decrease of stress in the coalescence regime. In particular, void coalescence can be sensitive to the void shape, which was considered to be fixed as a sphere in the present work. Deriving evolution equations for the void shape for strain hardening materials is a difficult task. Here, a phenomenological approach is considered in order to control the decrease of the stress during void coalescence. In the model presented in Section 3.2, the flow stress for void coalescence noted $\sigma_{*}^{g}$ was considered constant once coalescence has set on. Approaches to account for hardening were proposed by Scheyvaerts et al. (2011) and Vishwakarma and Keralavarma (2019). Here a formulation is proposed in order to be able to control the acceleration of porosity and stress decrease in the spirit of the work of Brepols et al. (2017). The coalescence flow stress now writes

$$
\sigma_{*}^{g} \leftarrow \sigma_{*}^{g}+\omega\left(1-\exp \left(-\frac{\gamma^{c}}{\beta}\right)\right)
$$

where $\omega$ and $\beta$ are additional material parameters that can be adjusted to control the rate of void coalescence. Their role will be further discussed on the basis of material point simulations in Section 4.3. 
Table 1

Summary of equilibrium equations, state laws and evolution equations.

\begin{tabular}{|c|c|c|}
\hline Equilibrium equations & State laws & Evolution equations \\
\hline $\operatorname{Div} \underset{\sim}{S}=\underline{\mathbf{0}} \quad \forall \underline{X} \in D_{0}$ & $\underset{\sim}{\boldsymbol{\Pi}^{e}}=\underset{\sim}{\boldsymbol{C}}: \underset{\sim}{\boldsymbol{E}_{G L}^{e}}$ & $\underset{\sim}{\dot{\boldsymbol{E}}}=\underset{\sim}{\dot{\boldsymbol{F}}} \cdot \underset{\sim}{\boldsymbol{F}}{ }^{-1} \cdot \underset{\sim}{\boldsymbol{E}}-\underset{\sim}{\boldsymbol{E}} \cdot\left(\sum_{k \in\{s ; c\}} \dot{\gamma}^{k} \boldsymbol{N}_{*}^{k}\right)$ \\
\hline 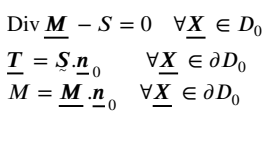 & $\begin{array}{l}\underline{\boldsymbol{M}}=A \underline{\boldsymbol{K}}_{\chi} \\
S=\Delta_{\chi}-\mu_{\chi} \gamma_{c u m}\end{array}$ & $\begin{array}{l}\dot{\gamma}^{k}=\dot{\gamma}_{0}\left\langle\frac{\tilde{\phi}^{k}}{\tau_{0}(1-f)}\right\rangle^{n} \text { with } k \in\{s ; c\} \text { and } s=\llbracket 1, N \rrbracket \\
\dot{r}^{s}=g_{s}\left(r_{s}\right)\left|\dot{\gamma}^{s}\right| \text { with } s=\llbracket 1, N \rrbracket \\
\dot{\gamma}_{\text {cum }}=\sum_{k \in\{s ; c\}}\left|\dot{\gamma}^{k}\right| \\
\dot{f}=(1-f) \operatorname{tr}\left(\underset{\sim}{\dot{P}} . P_{\sim}^{-1}\right)\end{array}$ \\
\hline
\end{tabular}

\subsection{Evolution equations}

The evolution of the elastic part of the deformation gradient $\underset{\sim}{\boldsymbol{E}}$ is deduced from the total and plastic deformation rates and is written as

$$
\underset{\sim}{\dot{\boldsymbol{E}}}=\underset{\sim}{\dot{\boldsymbol{F}}} \cdot \underbrace{\boldsymbol{F}}_{\sim} \cdot \underset{\sim}{\boldsymbol{E}}-\underset{\sim}{\boldsymbol{E}} \cdot\left(\sum_{k \in\{s ; c\}} \dot{\gamma}^{k} \boldsymbol{N}_{*}^{k}\right)
$$

The evolution law for porosity used in the context of ductile fracture of porous materials usually writes

$$
\dot{f}=(1-f) \operatorname{tr}\left(\underset{\sim}{\dot{P}} \cdot \stackrel{\sim}{P}^{-1}\right)
$$

Note that this equation holds during void growth as well as during void coalescence. Eq. (48) can be time-integrated and leads to the following explicit expression of the porosity

$$
f=1-\frac{1-f_{0}}{\operatorname{det}(\underset{\sim}{\boldsymbol{P}})}
$$

where $f_{0}$ denotes the initial void volume fraction.

In addition, a viscoplastic flow rule is adopted for each deformation mechanism (void growth mechanisms and void coalescence mechanism). In that way evolution of the plastic slip variables $\dot{\gamma}^{s}$ and $\dot{\gamma}^{c}$ are indistinguishably governed by the following Norton type flow rule

$$
\dot{\gamma}^{k}=\dot{\gamma}_{0}\left\langle\frac{\tilde{\phi}^{k}}{\tau_{0}^{k}(1-f)}\right\rangle^{n} \quad k \in\{s ; c\}
$$

where $\dot{\gamma}_{0}$ and $n$ are materials parameters controlling the rate sensitivity of the material behaviour. The Macaulay brackets are defined such that $\langle\bullet\rangle=\max (\bullet, 0)$. Distinct values of these viscosity parameters can be selected for growth and coalescence, if necessary. Note that the term $(1-f)$ at the denominator accounts for the fact that the slip rates $\dot{\gamma}^{k}$ are power conjugate to the effective matrix stresses $\tau_{*}^{s}$ and $\sigma_{*}^{c}$ as depicted in Eq. (42).

\subsection{Summary of constitutive equations and material parameters}

Equilibrium equations, state laws and evolution equations are summarized in Table 1. Four categories of material parameters can be identified:

1. Material parameters for elasto-viscoplasticity

2. Initial void characteristics

3. Porous criteria GTN-like parameters

4. Strain gradient plasticity parameters

They are listed in Table 2 with their corresponding unit and signification.

\section{Model implementation, calibration and assessment}

\subsection{Material behaviour}

In this study a face-centered cubic (FCC) crystal composed of $N=12$ octahedral slip systems of the $\{111\}\langle 110\rangle$ family is considered. The hardening behaviour used is a standard dislocation density based law following Kubin et al. (2008). The critical resolved shear stress of a given system $s$ is composed of a thermal component due to lattice friction $\tau_{0}$ and an athermal component $R_{s}$ due to dislocations interactions

$$
\tau_{c}^{s}=\tau_{0}+R_{s}=\tau_{0}+\mu \sqrt{\sum_{u=1}^{N} a^{s u} r^{u}}
$$


Table 2

Summary of material parameters involved in the strain gradient porous crystal plasticity model.

\begin{tabular}{clll}
\hline Category & Parameter & Unit & Signification \\
\hline \multirow{4}{*}{1} & $C_{i j k l}$ & $\mathrm{MPa}$ & Elastic moduli \\
& $\tau_{0}$ & $\mathrm{MPa}$ & Initial critical resolved shear stress \\
& $\dot{\gamma}_{0}$ & $\mathrm{~s}^{-1}$ & Reference slip rate \\
& $n$ & - & Viscosity exponent \\
& $\psi_{h} / R_{s}, R_{c u m}$ & $\mathrm{MPa}$ & Hardening potential/Hardening functions \\
& $g_{s}$ & - & Hardening variables evolution functions \\
& $f_{0}$ & - & Initial porosity \\
& $\lambda_{c}^{0} / \chi_{0}$ & - & Initial cell aspect ratio/intervoid distance \\
& $q_{1}, q_{2}, \alpha$ & - & Void growth GTN-like parameters \\
4 & $q_{1}^{c}, q_{2}^{c}, f_{R}, f_{u}^{*}, q_{\chi}$ & - & Void coalescence GTN-like parameters \\
& $A$ & $\mathrm{MPa} \cdot \mathrm{mm}^{2}$ & Strain gradient modulus \\
& $\mu_{\chi}$ & $\mathrm{MPa}$ & Penalization modulus \\
\hline
\end{tabular}

Table 3

Numerical values of material parameters corresponding to a $316 \mathrm{~L}$ stainless steel.

\begin{tabular}{llllll}
\hline$C_{11}$ & $C_{12}$ & $C_{44}$ & $\tau_{0}$ & $n$ & $\gamma_{0}$ \\
\hline $200 \mathrm{GPa}$ & $136 \mathrm{GPa}$ & $105 \mathrm{GPa}$ & $88 \mathrm{MPa}$ & 15 & $10^{14} \mathrm{~s}^{-1}$ \\
\hline$\mu$ & $G_{c}$ & $\kappa$ & $r_{0}^{s}$ & $b^{s u}(s \neq u)$ & $b^{u u}$ \\
\hline $65.6 \mathrm{GPa}$ & 10.4 & 42.8 & $5.38 \times 10^{-11}$ & 1 & 0 \\
\hline$a_{1}$ & $a_{2}$ & $a_{3}$ & $a_{4}$ & $a_{5}$ & $a_{6}$ \\
\hline 0.124 & 0.124 & 0.07 & 0.625 & 0.137 & 0.122 \\
\hline$f_{0}$ & $\lambda_{c}^{0}$ & $q_{1}$ & $q_{2}$ & $\alpha$ & $q_{1}^{c}$ \\
\hline $1 \%$ & 1 & 1.471 & 1.325 & 6.456 & 1 \\
\hline$q_{2}^{c}$ & $f_{R}$ & $f_{u}^{*} \stackrel{\text { def }}{\equiv} 1 / q_{1}$ & $\mu_{\chi}$ & $A$ & $\ell_{c} \equiv \sqrt{A / \tau_{0}}$ \\
\hline 1.5 & 0.35 & 0.67 & $500 \mathrm{MPa}$ & $1 \mathrm{~N}$ & $106.6 \mu \mathrm{m}$ \\
\hline
\end{tabular}

where $\mu$ is the shear modulus and $a^{s u}$ a matrix describing interactions between dislocations. $r^{u}$ denotes the adimensional dislocation density $\left(r^{u} / b^{2}=\rho^{u}\right.$ is the usual dislocation density, i.e. the length of dislocation lines per unit volume, $b$ being the norm of the dislocation Burgers vector $\underline{\boldsymbol{b}}$ ). The evolution of dislocation densities is given by the following rate equations

$$
\dot{r}^{s}=\left|\dot{\gamma}^{s}\right|\left(\frac{1}{\kappa} \sqrt{\sum_{u=1}^{N} b^{s u} r^{u}}-G_{c} r^{s}\right)
$$

Numerical values of material parameters corresponding to a 316L stainless steel and used throughout this work are listed in Table 3.

\subsection{Validation of the coalescence criterion}

\subsubsection{Single crystal porous unit-cell simulations}

In order to validate the capability of the criterion given by Eq. (34) to detect onset of coalescence the following procedure is proposed. Porous unit-cell finite element simulations are performed for several crystal orientations, stress triaxiality ratios and hardening behaviour. Onset of void coalescence can then be identified in each unit-cell simulation. Finally, the value of $\sigma_{I}$ at onset of coalescence $\left(\sigma_{I}^{\text {num }}\right)$ is compared to the theoretical value $\left(\sigma_{I}^{\text {th }}\right)$ predicted by the proposed criterion Eq. (26). The boundary conditions applied to the single crystal porous unit-cells are described in Appendix.

Cubic unit-cells composed of a FCC single crystal matrix material and a centered spherical void are considered. The initial porosity is equal to $1 \%$. Two sets $\mathcal{O}_{1}$ and $\mathcal{O}_{2}$ of crystal orientations (given with respect to the unit-cell lattice periodicity directions $\underline{X}_{1}-\underline{X}_{2}$ $-\underline{\boldsymbol{X}}_{3}$ ) are considered

$$
\mathcal{O}_{1}=\left\{\begin{array}{l}
{[100]-[010]-[001]} \\
{[\overline{1} 25]-[1 \overline{2} 1]-[210]} \\
{[111]-[\overline{2} 11]-[0 \overline{1} 1]} \\
{[110]-[\overline{1} 10]-[001]}
\end{array}\right\} \quad \mathcal{O}_{2}=\left\{\begin{array}{l}
{[210]-[\overline{1} 20]-[001]} \\
{[\overline{1} 25]-[0 \overline{5} 2]-[2925]} \\
{[100]-[01 \overline{1}]-[011]} \\
{[100]-[02 \overline{1}]-[012]}
\end{array}\right\}
$$

For the first set $\mathcal{O}_{1}$ unit-cell simulations were performed in Ling et al. (2016) with the material parameters corresponding to a 316L stainless steel listed in Table 3. To broaden the range of considered orientations, in this study additional unit-cell simulations are performed for all orientations in $\mathcal{O}_{1}$ and $\mathcal{O}_{2}$ with the same materials parameters but $a^{s u}=0$, i.e. in the absence of hardening. 


\subsubsection{Coalescence onset}

Onset of coalescence by intervoid necking is characterized by the transition to a uniaxial straining mode (pure extension) during which plastic deformation localizes in the intervoid ligament. This transition is marked by the saturation of transverse (in the coalescence plane) deformation. In other words, if the coalescence plane is normal to $\underline{X}_{1}$, the components $\bar{F}_{22}$ and $\overline{\boldsymbol{F}}_{33}$ of the deformation gradient will saturate. Therefore, for an increment $\Delta \overline{\boldsymbol{F}}$ of the macroscopic deformation gradient, onset of void coalescence can be detected when the ratios $\Delta \bar{F}_{22} / \Delta \bar{F}_{11}$ and $\Delta \bar{F}_{33} / \Delta \tilde{\bar{F}}_{11}$ become lower than an arbitrary low value. Onset of coalescence will be considered when these two ratios are simultaneously lower than $5 \%$. During the post-processing of a unit-cell simulation, the lowest deformation $\bar{F}_{11}^{c}$ at which this condition is met, is considered as the onset of coalescence and the maximum principal Cauchy stress at coalescence is recorded as $\sigma_{I}^{\text {num }}=\bar{\sigma}_{11}\left(\bar{F}_{11}^{c}\right)$. At the same time the value of $C_{f} \sigma_{*}^{c}$ is computed. For that purpose, the form of $C_{f}$ derived for isotropic materials in Thomason (1985), Benzerga et al. (1999) and Pardoen and Hutchinson (2000) is adopted

$$
C_{f}(\chi, W)=\left(1-\chi^{2}\right)\left(0.1\left(\frac{1-\chi}{\chi W}\right)^{2}+1.2 \sqrt{\frac{1}{\chi}}\right)
$$

where $\chi$ and $W$ respectively represent effective normalized intervoid distance and void aspect ratio. The limit load formula in Eq. (54) has been thoroughly revisited in the recent literature (Benzerga and Leblond, 2014; Morin et al., 2015). In particular, a remedy to the singularity in the limit of a penny-shape crack $(W \rightarrow 0)$ was proposed (Hure et al., 2016; Torki et al., 2017). In addition, the validity of Eq. (54) for porous single crystals is not straightforward, because it was derived in the context of isotropic plasticity. A derivation of the limit load of a porous unit-cell was extended recently to the crystal plasticity framework (Hure, 2019). Yet, for the sake of simplicity, it remains interesting to evaluate the validity of the limit load in Eq. (54) in presence of plastic anisotropy. Extensions to possibly more accurate and more complex coalescence criteria is left for further studies. Thomason's original criterion was derived on an orthorhombic unit-cell containing an orthorhombic void. However the orthorhombic unit-cell considered here contains a spherical void. As discussed by Scherer and Hure (2019) a parameter $q_{\chi}$ can be introduced in order to account for this difference. In that way, $C_{f}$ is still expressed as in Eq. (54), but the intervoid ligament ratio $\chi$ defined in Eq. (33) is replaced by

$$
\chi \leftarrow q_{\chi} \chi
$$

A straightforward geometrical analysis shows that the transition from an orthorhombic void to a spherical void yields to $q_{\chi}=\sqrt{\pi / 6}$. Yet, $q_{\chi}$ could also be considered as an additional coefficient to be identified in the same vein as $q_{1}$, $q_{2}$ and $\alpha$ for the void growth model. For the sake of simplicity, the analytical value of $\sqrt{\pi / 6}$ is used in the present study. To estimate $\chi$ and $W$ it is assumed for simplicity that the initially cubic cell remains orthorhombic and that the initially spherical voids remain ellipsoidal when deforming. Although these assumptions might be crude for highly deformed cells at coalescence, especially at low triaxialities, it is the simplest way to obtain estimates of $\chi$ and $W$. With these assumptions a deformed unit-cell is characterized by $L_{1}, L_{2}$ and $L_{3}$ which are respectively computed by following the displacements of the nodes initially located at the middle of each face of the unit-cell. The void is characterized by three semi-axes $r_{1}, r_{2}$ and $r_{3}$ which are respectively computed by following the displacements of the nodes initially located at the intersection of the void with the three major axis of the cube (void poles). The geometrical parameters $\chi$ and $W$ are then computed as follows

$$
\chi=\sqrt{\chi_{2} \chi_{3}}=\sqrt{\frac{2 r_{2}}{L_{2}} \frac{2 r_{3}}{L_{3}}} \quad W=2 \frac{W_{12} W_{13}}{W_{12}+W_{13}}=2 \frac{\frac{r_{1}}{r_{2}} \frac{r_{1}}{r_{3}}}{\frac{r_{1}}{r_{2}}+\frac{r_{1}}{r_{3}}} \quad \lambda_{c}=\frac{L_{1}}{\sqrt{L_{2} L_{3}}}
$$

which boil down to their usual definition when the void is a sphere and the cell a cube $(\chi=2 R / L$ and $W=1)$. Yet, the choice made in Eq. (56) to define $\chi, W$ and $\lambda_{c}$ is not unique. Neglecting viscous effects, the value of $\sigma_{*}^{c}$ should be equal to $\sigma_{*}^{g}$ at onset of coalescence, hence $\sigma_{*}^{g}$ is computed by solving Eq. (36) where $q_{1}^{c}=1.5$ and $q_{2}^{c}=1$ are chosen. The theoretical coalescence stress is therefore $\sigma_{I}^{t h}=C_{f}(\chi, W) \sigma_{*}^{g}$.

In Fig. 1, the numerical coalescence stresses are plotted against the theoretical coalescence stresses for all the simulations for which coalescence was attained. If the criterion were to be exact the points would be located on the ' $y=x^{\prime}$ bisector. As it is only an approximation the points may not exactly lay on this line. Almost all the predicted coalescence stress values are less than $\pm \% 20$ apart from the values predicted by the unit-cell simulation. It can be seen that the criterion is capable of well predicting the coalescence onset with or without hardening of the matrix material surrounding the void. A more refined tuning of the function $C_{f}$ in Eq. (56) and the parameters in Eq. (36) could lead to a more precise prediction of coalescence onsets. This is however beyond the scope of the present study.

\section{3. $f^{*}$-type coalescence vs void coalescence mechanism}

Several variations of the model presented above are compared in this section. Ductile fracture by void growth only is compared to ductile fracture by void growth and void coalescence, with either the $f^{*}$-type treatment of coalescence or its yield mechanism based treatment. A single hexahedral element with eight nodes and reduced integration with one Gauss point is loaded with a constant stress triaxiality similarly to the periodic porous unit-cells in Section 4 . Triaxialities of 1 and 3 are applied. Three different crystal orientations are considered and correspond to the three first orientations in set $\mathcal{O}_{1}$. An initial porosity of $1 \%$ is considered and numerical values of other material parameters used are listed in Table 3. Since a single Gauss point is used, no gradients of 


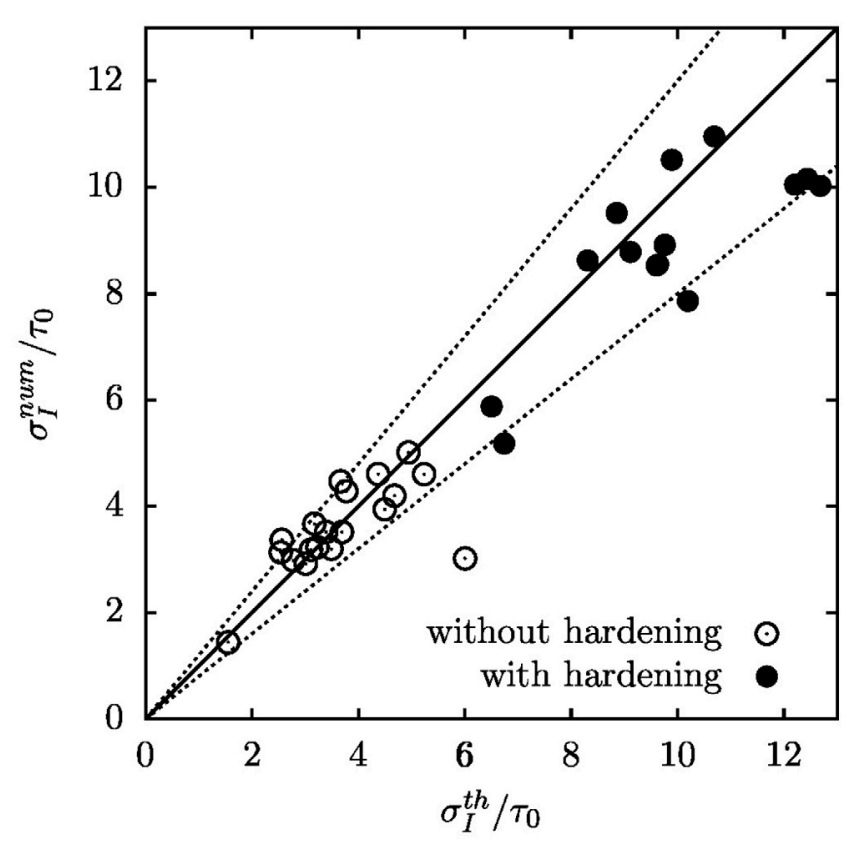

Fig. 1. Numerically computed principal Cauchy stress in unit-cell simulations $v s$ theoretical prediction of the principal Cauchy stress at onset of coalescence. Dashed lines represent the $\pm 20 \%$ error from the case were the theoretical prediction matches the numerical value.

accumulated plastic slip can form and therefore the non-local moduli $A$ and $\mu_{\chi}$ do not influence the results presented hereafter. Stress $v s$ strain responses and porosity evolution are plotted in Fig. 2 . Solid lines correspond to cases at a triaxiality $T=1$ and dashed lines to cases at $T=3$. Reference behaviours of the pristine void-free single crystal are plotted in black. The prediction of the model with void growth mechanisms only, i.e. without accounting for void coalescence, is plotted in the same graphs. The respective curves of $f^{*}$-type coalescence and void coalescence mechanism depart from one another only once coalescence sets on. In addition, since the same coalescence criterion is used for $f^{*}$-type coalescence and void coalescence mechanism, simulations accounting for coalescence start deviating from the void growth model at the same moment. When only void growth mechanisms are accounted for, stress and porosity evolution with respect to strain remain smooth all the way until fracture. However, when coalescence is taken into account, a corner appears at onset of void coalescence. That corner marks an acceleration of porosity increase which simultaneously triggers a sharp stress drop. In all cases presented in Fig. 2 but one, the acceleration of porosity increase is more pronounced with the void coalescence mechanism than with the $f^{*}$-type treatment of coalescence. As a consequence, stresses also drops faster.

The respective influence of parameters $\omega$ and $\beta$ in the coalescence induced hardening Eq. (46) is brought to light in Fig. 3 in which both parameters were independently varied. $\omega$ is taken in the range 1.5 to $100 \mathrm{GPa}$, while $\beta$ is in the range 0.1 to 10 . In the present example the coalescence flow stress $\sigma_{*}^{g}$ was equal to $615 \mathrm{MPa}$. Increasing $\omega$ results in a slower porosity growth and stress softening. On the contrary increasing $\beta$ has opposite effects, namely a faster porosity augmentation and a sharper stress drop. Fig. 4 shows how $\omega$ affects the void growth and coalescence inelastic variables after coalescence. As $\omega$ increases, void growth plastic variables increase more and more in the coalescence regime $\left(\exists s \dot{\gamma}^{s}>0\right)$, whereas for $\omega=0$ void growth mechanisms are completely inactive in the coalescence regime $\left(\forall s \dot{\gamma}^{s}=0\right)$. In contrast, as $\omega$ increases, the contribution of void coalescence $\gamma^{c}$ to the inelastic activity decreases in the post-coalescence regime. The role of Eq. (46) is thus to introduce strain hardening associated to the void coalescence mechanism. In that way, the softening rate can be calibrated from experiments or computational unit-cell results.

\subsection{Numerical aspects}

The model presented in previous sections was discretized using an Euler-backward (implicit) scheme and implemented in the finite element software Z-set (Besson and Foerch, 1998). Details on the finite element implementation of the Lagrange multiplier formulation were described in Scherer et al. (2020). In the subsequent finite element simulations, 20-node brick finite elements are used. Quadratic shape functions for displacement degrees of freedom (DOF) and linear shape functions for the Lagrange multiplier $\lambda$ and micro-slip $\gamma_{\chi}$ DOF are used. Reduced integration is performed by using eight Gauss points per element.

Highly damaged elements are suppressed from the finite element mesh during the computation. An element is considered as being "broken" when at least half of the Gauss points (4 out of 8 ) satisfy at least one of the following criteria

$$
\chi>(1-\varepsilon) \quad \text { or } f>(1-\varepsilon) f_{R} \text { or } \quad E_{G L, I}>E_{G L, I}^{c}
$$

where $\varepsilon$ is a small tolerance parameter set to $10^{-2}$ and $E_{G L, I}$ is the largest eigenvalue of the Green-Lagrange strain tensor $\underset{\sim}{\boldsymbol{E}}{ }_{G L}=(1 / 2)(\underset{\sim}{\boldsymbol{F}} \underbrace{\boldsymbol{F}}_{\sim}-\underset{\sim}{\mathbf{1}})$. The critical value $E_{G L, I}^{c}$ is set to 4. At the Gauss point level, if one of these criteria is met, the stress 


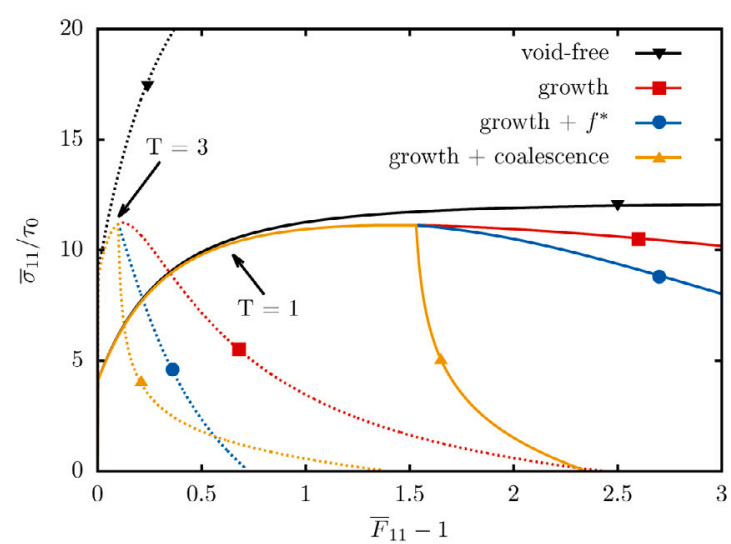

(a) $<100>$

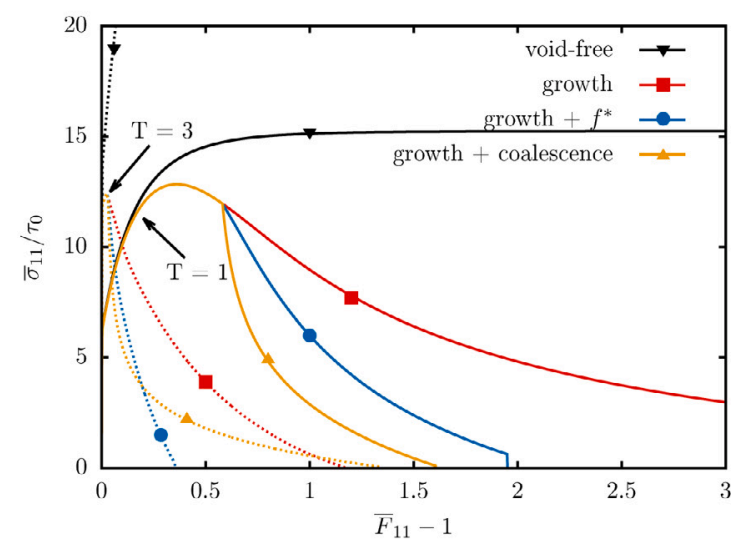

(c) $\langle 111>$

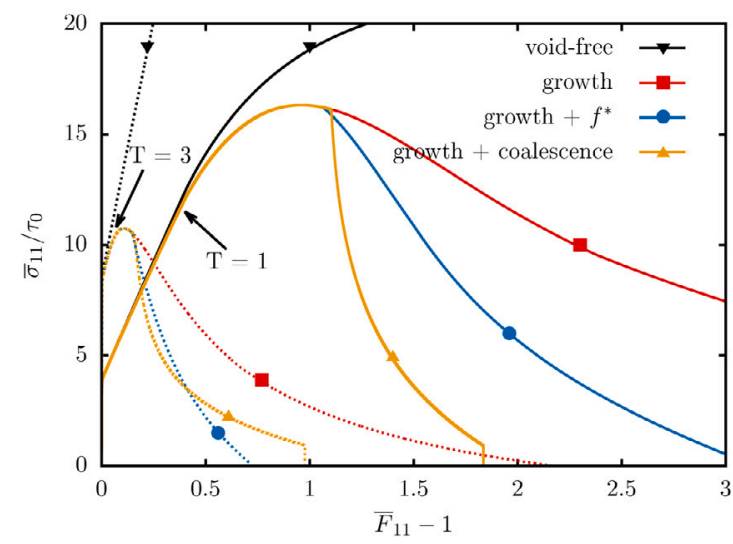

(e) $<125>$

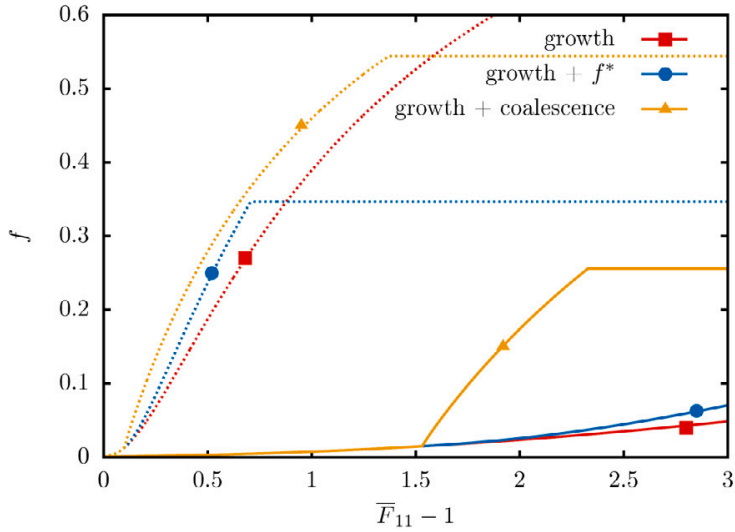

(b) $<100>$

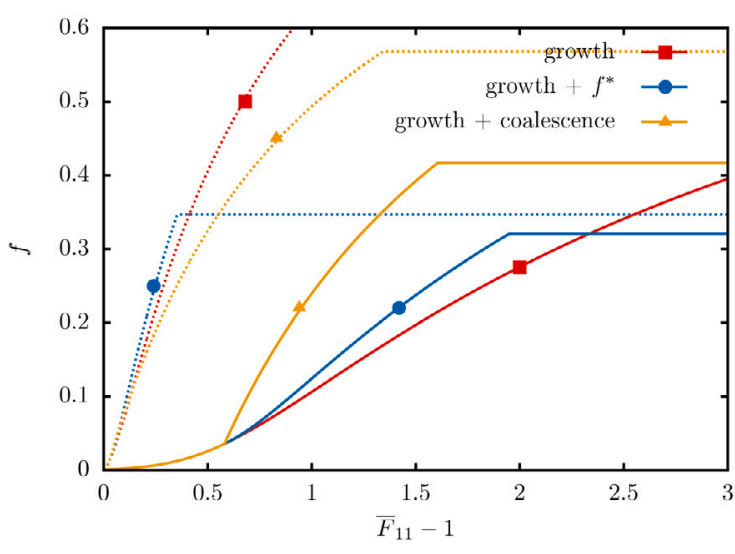

(d) $<111>$

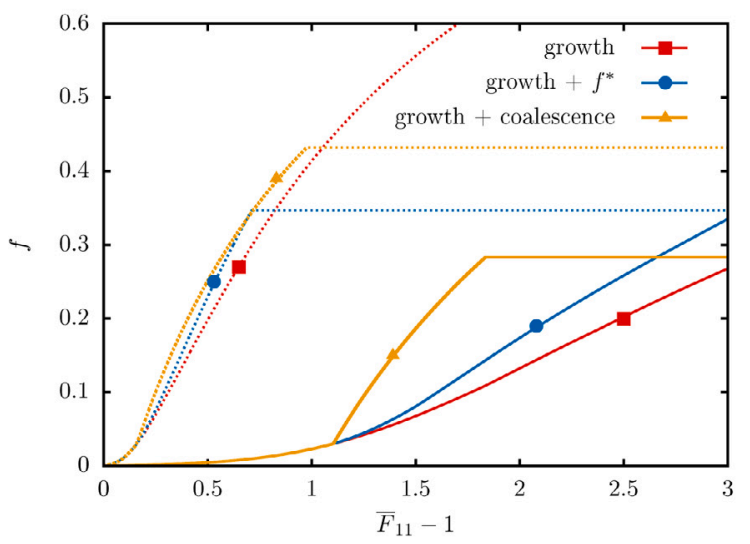

(f) $<125>$

Fig. 2. Stress-strain behaviour and porosity evolution at imposed stress triaxialities of 1 (solid lines) and 3 (dashed lines) on a single Gauss point with three different versions of the porous single crystal ductile failure model: void growth mechanisms only (red), void growth mechanisms and $f^{*}$-type coalescence (blue), and void growth and void coalescence mechanisms (orange). 


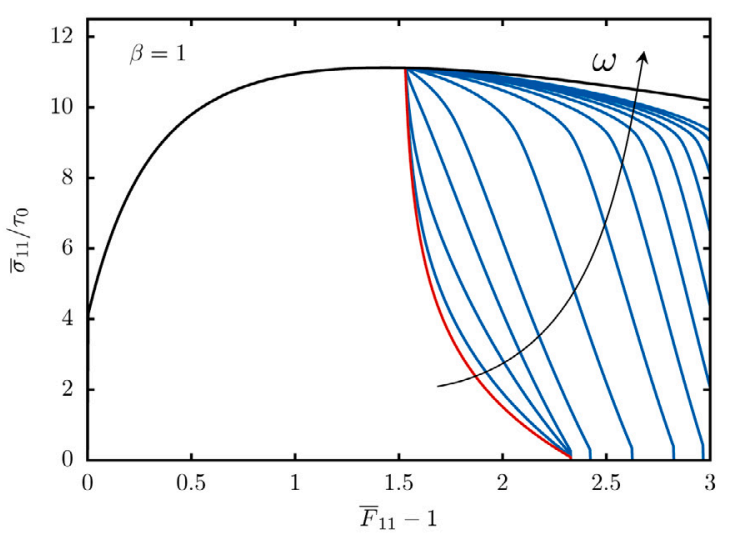

(a)

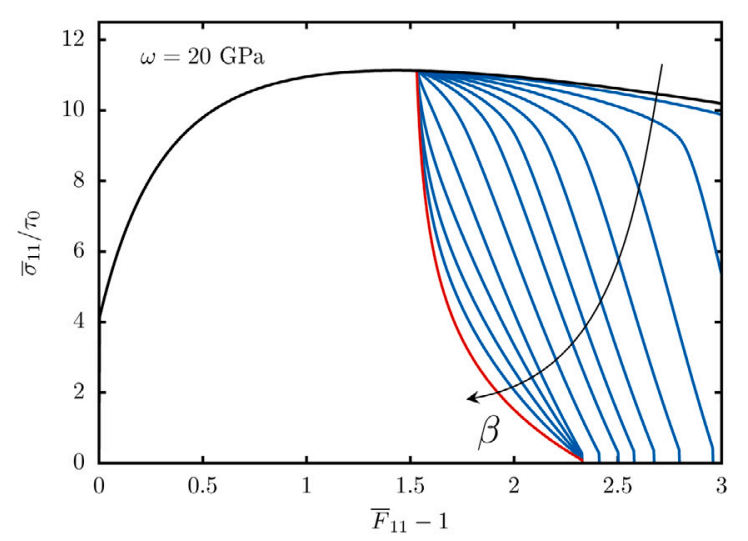

(c)

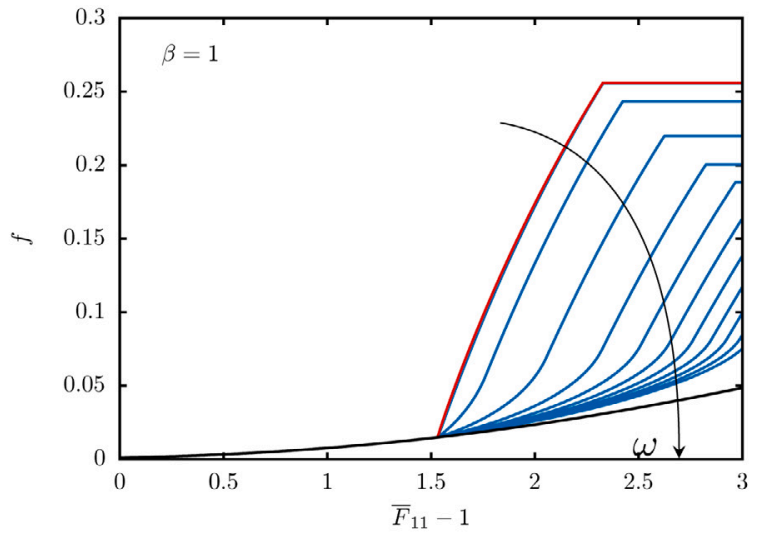

(b)

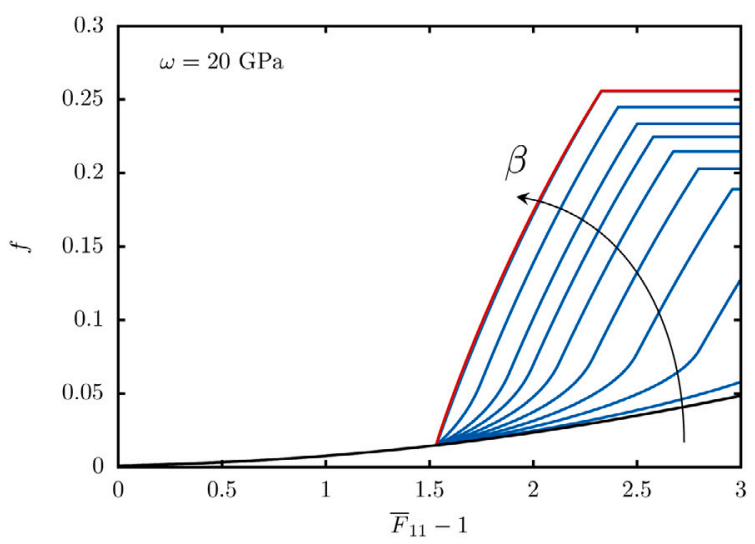

(d)

Fig. 3. Influence of material parameters $\omega$ and $\beta$ from Eq. (46) on the post-coalescence regime of the tensile stress and the porosity. $\omega$ varies from 1.5 to $100 \mathrm{GPa}$ and $\beta$ varies from 0.1 to $10 . \sigma_{*}^{g}=615 \mathrm{MPa}$ in this example.

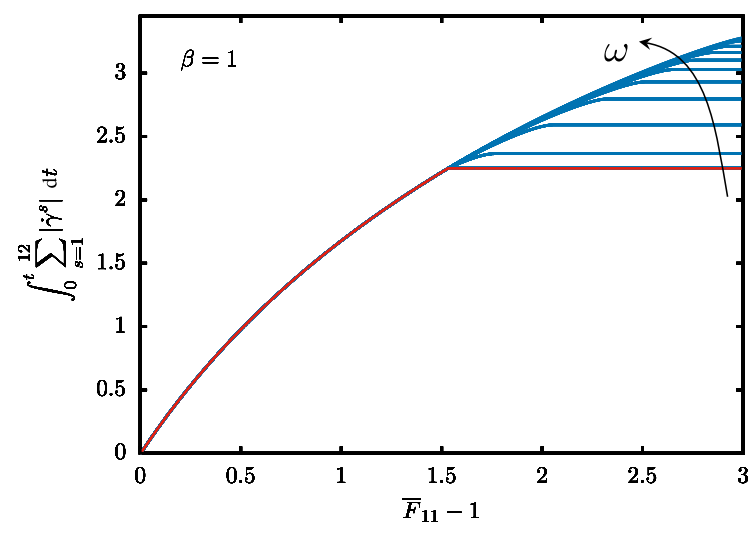

(a)

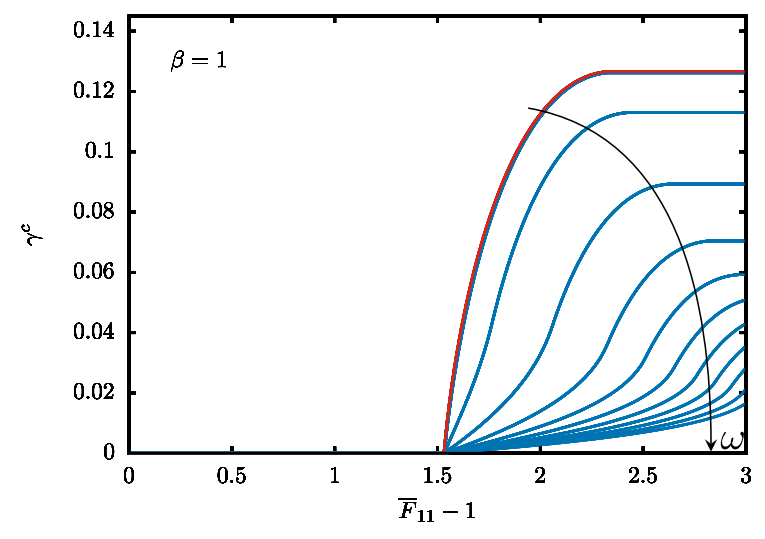

(b)

Fig. 4. Influence of material parameter $\omega$ on the post-coalescence regime of void growth and void coalescence plastic slip variables. $\omega$ varies from 1.5 to 100 $\mathrm{GPa}$ and $\beta$ is fixed to $1 . \sigma_{*}^{g}=615 \mathrm{MPa}$ in this example. 


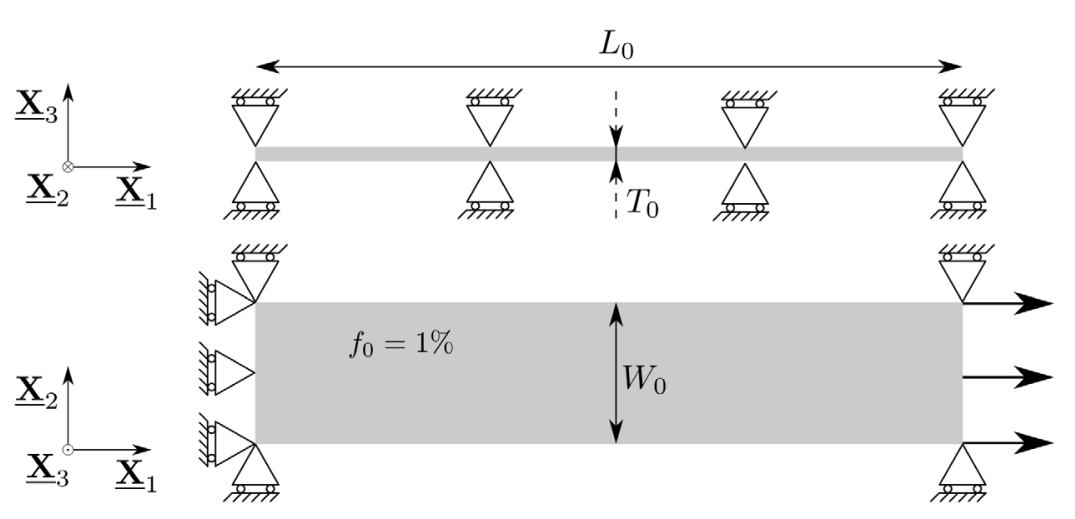

(a)

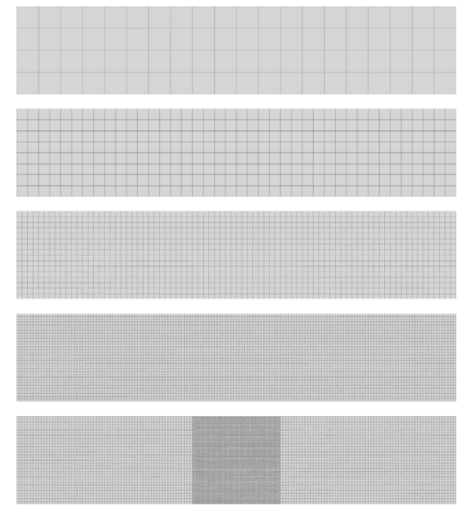

(b)

Fig. 5. (a) Sketch of the plate geometry and applied plane strain boundary conditions. (b) Finite element meshes used in the mesh convergence analysis. From top to bottom, meshes are respectively composed of $80,320,1280,5120$ and 13184 elements.

tensor is fixed to 0 and the tangent matrix is set to $10^{-6} \underset{\widetilde{\sim}}{\boldsymbol{C}}$, with $\underset{\widetilde{\sim}}{\boldsymbol{C}}$ the elasticity stiffness tensor. The last criterion on $E_{G L, I}$ is an $a d$ hoc criterion used in order to remove elements that are highly sheared or elongated. Elements of this kind are typically observed in regions of low stress triaxiality for which damage due to void growth remains very low. A possible way to get rid of this ad hoc criterion would consist in considering an effective porosity evolution law that accounts for shear induced damage (Nahshon and Hutchinson, 2008). Alternatively shear induced void coalescence could also be considered (Torki and Benzerga, 2018). This is however out of the scope of the present study and is left for a future work.

\subsection{Mesh convergence analysis}

A mesh convergence analysis is carried out in order to validate the regularization capacity of the model. A thin rectangular plate of initial length $L_{0}$, width $W_{0}=L_{0} / 5$ and thickness $T_{0}=W_{0} / 10$, as depicted in Fig. 5, is loaded in plane strain tension by applying the following boundary conditions

$$
\begin{aligned}
U_{1}\left(X_{1}=0, X_{2}, X_{3}\right)=0 & U_{1}\left(X_{1}=L_{0}, X_{2}, X_{3}\right)=U_{1}(t) \\
U_{2}\left(X_{1}=0, X_{2}, X_{3}\right)=0 & U_{2}\left(X_{1}=L_{0}, X_{2}, X_{3}\right)=0 \\
U_{3}\left(X_{1}, X_{2}, X_{3}\right)=0 &
\end{aligned}
$$

The plate is discretized with $m \in\{80,320,1280,5120,13184\}$ finite elements. The most dense mesh thus totals 280683 displacement DOF and 53756 Lagrange multiplier and micro-slip DOF. The mesh convergence analysis is performed on a single crystal with the crystal directions [100], [010] and [001] initially oriented along the orthonormal basis vectors $\underline{X}_{1}, \underline{X}_{2}$ and $\underline{X}_{3}$ respectively. For the sake of simplicity of this benchmark example hardening is discarded. The critical resolved shear stress is therefore constant and equal to $\tau_{0}$ for all slip systems. Void coalescence is accounted for by using the effective porosity $f^{*}$ defined at Eq. (45) once the coalescence onset criterion is met as discussed in Section 3.2.2. Numerical values of material parameters are listed in Table 3.

In Fig. 6 the normalized engineering stress $F /\left(S_{0} \tau_{0}\right)$ is plotted against the normalized width extension $-\Delta W / W_{0}$. The predictions of the model without regularization in Fig. 6(a) are compared to the predictions with strain gradient regularization in Fig. 6(b). At small strains all meshes produce identical results regardless of regularization. In this regime gradients of plastic strain are absent hence no size effects due to gradients arise. At large strains the coarse meshes display spurious oscillations of stress and strain due to insufficient mesh refinement in the region of interest. Nevertheless, no convergence is attained when mesh size is decreased in the post-localization regime in the absence of gradient contributions. On the contrary, when regularization is active, the localization of plastic strain is balanced by gradient-induced hardening. Convergence of numerical stress-strain curves with respect to mesh density is thus achieved with the strain gradient plasticity model as expected.

Fig. 7 displays the fields of the normalized intervoid distance $\chi$ which is the relevant damage variable during coalescence. $\chi$ strongly localizes in the necked region for both conventional and strain gradient porous crystal plasticity. It can be noted that with a conventional plasticity theory, i.e. not accounting for strain gradient hardening, the most dense mesh displays the most localized damage field. When mesh size is decreased a smaller volume needs thus to be completely damaged to reach failure. This explains why less energy is required for failure when mesh size is decreased and the absence of convergence with mesh size reduction. In contrast, with the strain gradient regularization, the damage variable spans over a similar volume for the five different meshes. Therefore macroscopic stress-strain curves are nearly mesh-size independent. Even though the macroscopic stress $v s$ strain curves and the damage fields are almost mesh-size independent with the strain gradient plasticity model, the local field of the damage 


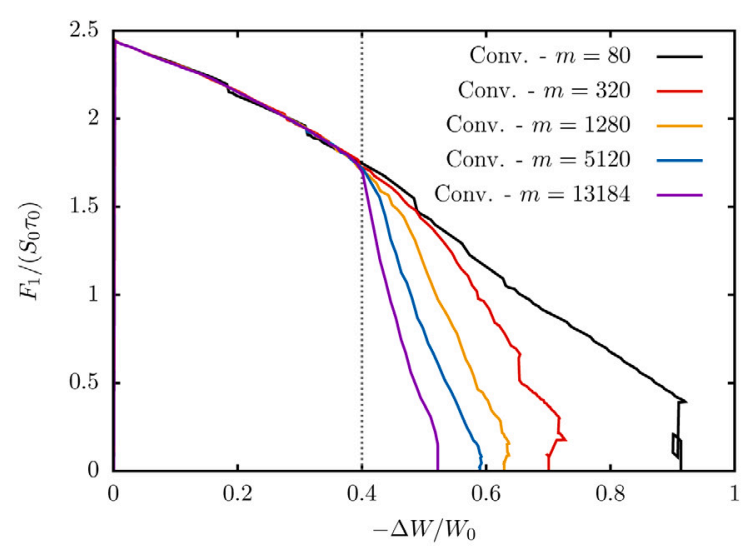

(a) Conventional

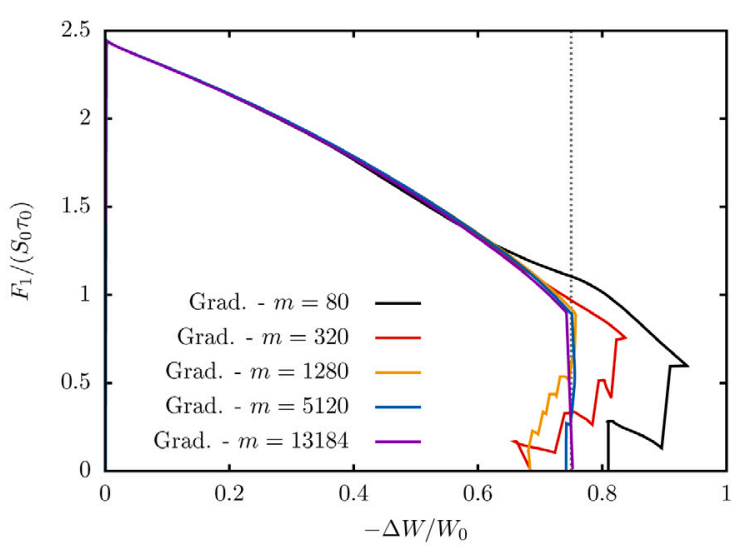

(b) Strain gradient $(A=0.1 \mathrm{~N})$

Fig. 6. Stress $v s$ normalized width reduction for conventional (a) and strain gradient (b) porous single crystal plasticity for the several mesh densities. Vertical dashed lines represent the strain level at which contours of damage variable $\chi$ are plotted in Fig. 7 .

variable $\chi$ still localizes ultimately to the thickness of one Gauss point. Eventually a one-element-thick discrete crack is nucleated in the middle of the specimen and propagates towards both edges of the specimen.

\section{Fracture toughness of porous crystals}

\subsection{Specimen geometry, mesh and boundary conditions}

A CT specimen geometry is meshed with a single element across the thickness (along $\underline{X}_{3}$ ). The notch front of the CT specimen is located at $X_{1}=a_{0}$. The notch is infinitely sharp, so that the notch radius is equal to zero. A vertical displacement $U_{2}^{G}(t)$ is applied to the nodes denoted by the letter $G$ in Fig. 8 (three nodes aligned along direction $\underline{X}_{3}$ through the thickness of the specimen). Plane strain conditions are imposed by enforcing $U_{3}=0$ to all nodes of the finite element mesh. Numerical values of material parameters used for the simulation of the CT specimen are listed in Table 3. The crack mouth opening displacement (CMOD) is defined as the vertical opening of the crack as shown in Fig. 8. In contrast to the plane strain tension simulations of previous section, hardening is now taken into account. The initial porosity $f_{0}$ is taken as $1 \%$ in most cases and as $0.1 \%$ in a few cases that will be indicated. Similarly the strain gradient modulus $A$ is taken equal to $1 \mathrm{~N}$ for most simulations apart from a few cases, specified later on, where it takes values equal to 0.1 and $10 \mathrm{~N}$. These values of $A$ can be related to an intrinsic material length scale by introducing the quantity $\ell_{c} \stackrel{\text { def }}{\equiv} \sqrt{A / \tau_{0}}$. The values of $0.1,1$ and $10 \mathrm{~N}$ for the parameter $A$ correspond to internal lengths $\ell_{c}$ equal to $31.6 \mu \mathrm{m}, 106.6 \mu \mathrm{m}$ and $316.2 \mu \mathrm{m}$ respectively. These lengths are to be compared to the specimen characteristic size which is of the order of $W=40 \mathrm{~mm}$. The size of elements within the refined region of the mesh is equal to $\ell_{0}=66.7 \mu \mathrm{m}$. The mesh size is therefore sufficient to resolve strain localization phenomena and obtain mesh size independent results with the strain gradient plasticity model. In the following, the conventional and strain gradient plasticity versions of the porous single crystal model are compared.

\subsection{Single crystal specimens}

Figs. 9 and 10 show the accumulated plastic slip fields in the vicinity of the notch front of the CT specimen for several crystal orientations. Results obtained with the conventional model are shown in Fig. 9 while those obtained with the strain gradient plasticity model are presented in Fig. 10. The crystal orientations inverse pole figures are shown in Fig. 9(g). In all these simulations, some extent of crack propagation was reached. With the conventional model, striking variations of crack paths are observed between different orientations. For two highly symmetric orientations 9 (c) and 9 (d) the crack propagates straight in the middle symmetry plane of the specimen. The highly symmetric orientation 9(a), the low symmetry orientation 9 (e) and the non-symmetric orientation 9(b) display crack paths that deviate from the middle plane. In the case of orientation 9(e) the crack starts to propagate out of the middle plane and later bifurcates back to an horizontal plane of propagation. Orientations 9 (f) displays the most serrated crack path. For this orientation the crack oscillates around the middle symmetry plane of the specimen.

As shown in Fig. 10, crack paths obtained with the strain gradient plasticity model remain closer to the middle plane than those obtained with the conventional model. Furthermore it is particularly clear from Figs. 9(a), 9(b) and 10(a), 10(b), that the strain gradient plasticity model predicts much smoother plastic strain fields than the conventional model. This less localized plastic activity could explain why crack paths predicted by the strain gradient plasticity model are less influenced by the crystal orientation. The strain gradient regularization indeed prevents intense strain localization from occurring along directions that are preferential for plastic slip. As a consequence, the role of the applied mechanical loading on the crack path evolution is emphasized at the detriment 

0.2
0.3
0.4
0.5
0.6
0.7
0.8
0.9

Conventional porous crystal plasticity $\left(\right.$ at $\left.-\frac{\Delta W}{W_{0}}=0.4\right) \quad$ Strain gradient porous crystal plasticity $\left(\right.$ at $\left.-\frac{\Delta W}{W_{0}}=0.75\right)$

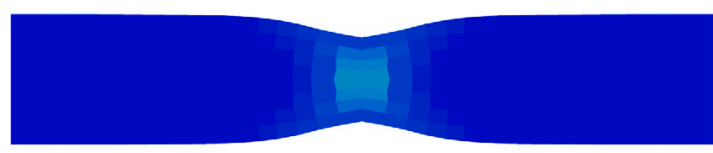

(a) $m=80$

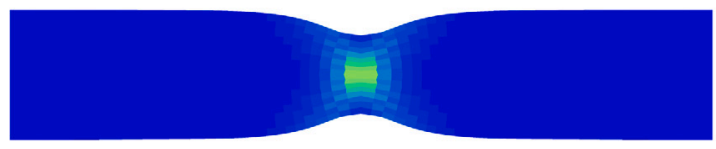

(c) $m=320$

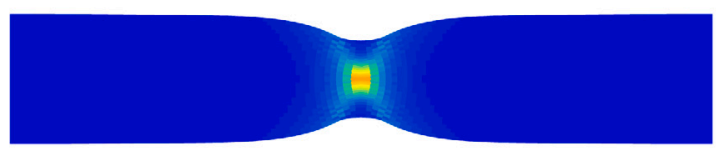

(e) $m=1280$

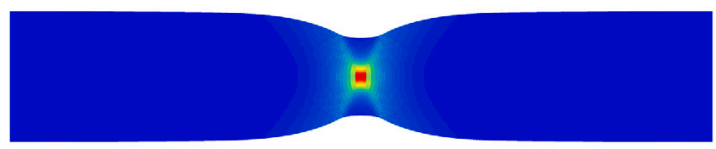

(g) $m=5120$

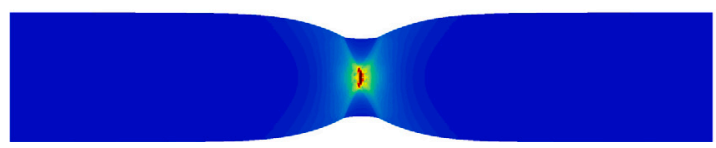

(i) $m=13184$

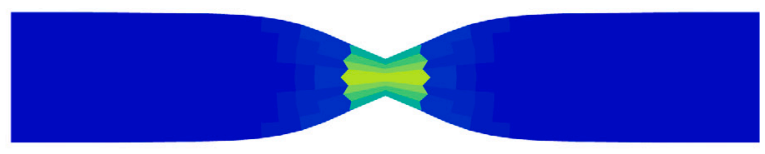

(b) $m=80$

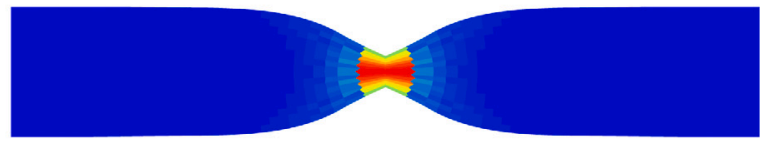

(d) $m=320$

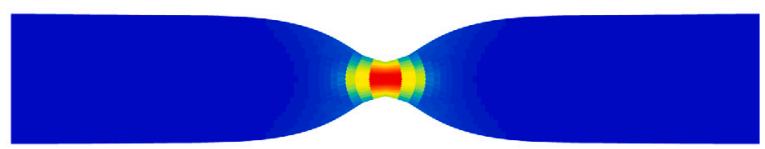

(f) $m=1280$

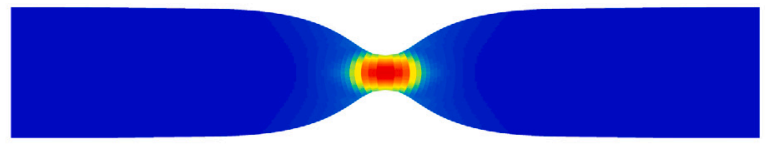

(h) $m=5120$

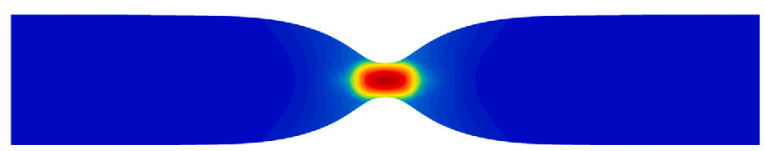

(j) $m=13184$

Fig. 7. Field of damage variable $\chi$ at $-\Delta W / W_{0}=0.4$ for conventional (left) and at $-\Delta W / W_{0}=0.75$ for strain gradient (right) porous single crystal plasticity for the several mesh densities.

of less influence from crystal orientation. Fig. 11 displays the curves of applied load $F$ at point $G$ against the CMOD. The predictions of the conventional model are shown in Fig. 11(a) and those of the strain gradient plasticity model in Fig. 11(b). It is recalled that loading curves obtained with the conventional porous model are mesh size dependent so that presented results are for a given mesh size. Yet, results with the conventional model already highlight the difference in terms of ductility that exist between different crystal orientations. Taking strain gradients into account postpones the crack propagation and the associated load decrease. As pictured in Fig. 10, damage is indeed smeared over several Gauss point layers, thus requiring a larger amount of energy to be supplied in order to drive the crack forward. Although still significant, the difference of the loading curves and ductility is smaller with the strain gradient plasticity model than with the conventional model. This observation echos the fact that the strain gradient plasticity model predicts less serrated and more similar crack paths between crystal orientations than the conventional model does.

The CT single crystal simulations were post-processed in order to determine the crack extension $\Delta a=a(t)-a_{0}$, where $a(t)$ is the crack length along $\underline{X}_{1}$ at time $t$. The plastic component $J_{p l}$ of the fracture toughness $J$ was computed according to the ASTM Standard E1820 (2017) which gives the following expression

$$
J_{p l}=\frac{\eta_{p l} A_{p l}}{B_{N} b_{0}}
$$

where $A_{p l}$ is the plastic part of the area located below the load vs CMOD curves, $B_{N}=66.7 \mu$ m is the net specimen thickness, $b_{0}=W-a_{0}=15.6 \mathrm{~mm}$ is the uncracked ligament length and $\eta_{p l}=2+0.522 b_{0} / W=2.20358$. Note that the crack growth correction proposed in ASTM Standard E1820 (2017) was not applied when computing $J_{p l}$. Fig. 12 shows the evolution of $J_{p l}$ with the crack 


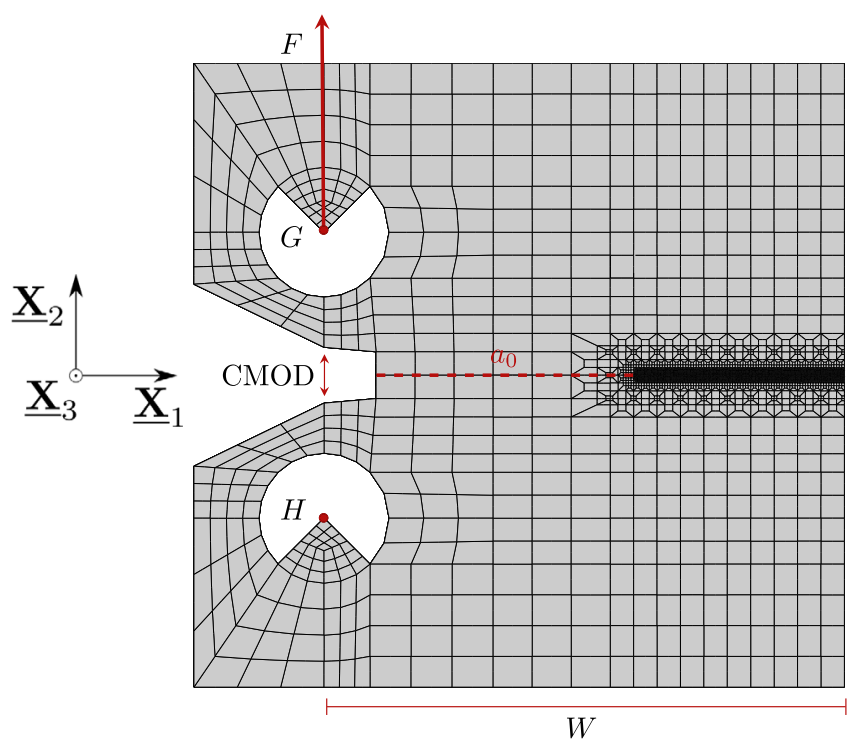

Fig. 8. Finite element mesh of a CT specimen geometry of width $W=40 \mathrm{~mm}$ with initial crack length $a_{0}=24.4 \mathrm{~mm}$ and applied load $F$. The mesh is composed of 544020 -node brick elements containing 8 Gauss points each. In the refined region of the mesh, elements are cubes of size $\ell_{0}=66.7 \mu \mathrm{m}$.

extension $\Delta a$ for the different single crystal specimens. The predictions of the conventional model are shown in Fig. 12(a) and those of the strain gradient plasticity model in Fig. 12(b). An important difference between crystal orientations is also observed. The less ductile orientations display a nearly flat evolution of $J_{p l}$ with the crack extension. On the contrary, orientations which display appreciable ductility show a fast increase of $J_{p l}$ with $\Delta a$. For all orientations, the strain gradient plasticity model predicts a larger value of $J_{p l}$ at initiation of crack propagation $(\Delta a>0)$ than the conventional model. In addition, the increase of $J_{p l}$ with $\Delta a$ is steeper with the strain gradient plasticity model.

In order to quantify simultaneously the effect of the void volume fraction and the internal length, [100]-[010]-[001] single crystal CT specimens with initial porosity $f_{0}=0.1 \%$ and $1 \%$ and with strain gradient modulus $A=0.1 \mathrm{~N}, 1 \mathrm{~N}$ and $10 \mathrm{~N}$ were simulated. The fields of damage variable $\chi$ defined at Eq. (33) for the specimen with the initial porosity of $1 \%$ are shown in Fig. 13. It can be noticed that even a very small internal length (e.g. $A=0.1 \mathrm{~N}$ corresponding to $\ell_{c}=31.6 \mu \mathrm{m}$ ) can significantly affect the crack path. The mesh size is indeed small enough to capture strain gradient effects at this scale. For the conventional model's prediction in Fig. 13(a) and the strain gradient prediction with $A=0.1 \mathrm{~N}$ in Fig. 13(b), the crack paths are not straight. Nevertheless the conventional model displays a crack initially bifurcating towards the bottom of the specimen, while the strain gradient plasticity model leads to a crack propagating towards the top of the specimen. As the internal length increases the crack path becomes straight (see Figs. 13(c) and 13(d)). Simulations with the lower initial porosity of $0.1 \%$ (not shown here for conciseness) display a straight crack path whatever the internal length, even for the conventional model. A possible reason for this observation is that a large initial porosity allows to reach sooner the critical stress for the onset of coalescence. Regions located out of the horizontal symmetry plane can hence undergo coalescence more easily and lead to crack bifurcation. However, it cannot be excluded that the bifurcation observed with the conventional model and the strain gradient plasticity model at very small internal length is not an artefact due to the dependency to the finite element discretization.

For the small internal length, the damage variable does not spread much around the crack lips and front. In contrast, the larger internal length leads to damage fields smeared over multiple rows above and below the crack lips, but also smeared over multiple columns of elements ahead of the notch front. It is important to recall that the smoothing of the damage variable profile observed here is an indirect result of the strain gradient regularization of the accumulated plastic slip variable $\gamma_{\text {cum }}$. The damage variable is not explicitly regularized in this model. In the context of brittle fracture the damage variable is often used as the variable bearing gradient effects (Aslan et al., 2011; Lindroos et al., 2019). Recently Lindroos et al. (2020) compared brittle fracture models based on the regularization of accumulated slip, or accumulated damage or an additive combination of both. In the context of ductile fracture, a damage variable was used for regularization for instance in Reusch et al. (2003), Ramaswamy and Aravas (1998) and Håkansson et al. (2006), which was either the porosity or the effective porosity. However, as already discussed by Nguyen et al. (2020), at the final stage prior to failure, non-local models based on a damage indicator must satisfy that local and non-local variables eventually coincide. Decreasing internal lengths were considered in Poh and Sun (2017) to overcome this shortcoming.

Fig. 14 shows the load $v s$ CMOD and $J_{p l} v s \Delta a$ curves for the different internal lengths and initial porosities. As expected, as the strain gradient modulus increases, the CT specimen displays an increased ductility characterized by a larger maximum load and a slower load decrease in Fig. 14(a). This behaviour translates into a larger value of $J_{p l}$ at onset of crack propagation ( $\left.\Delta a>0\right)$ and a steeper rise of $J_{p l}$ with the crack extension in Fig. 14(b). In addition, a smaller initial porosity leads also to a greater ductility. The conventional model with an initial porosity of $f_{0}=0.1 \%$ predicts a quasi-brittle behaviour similarly to what predicts the same model 


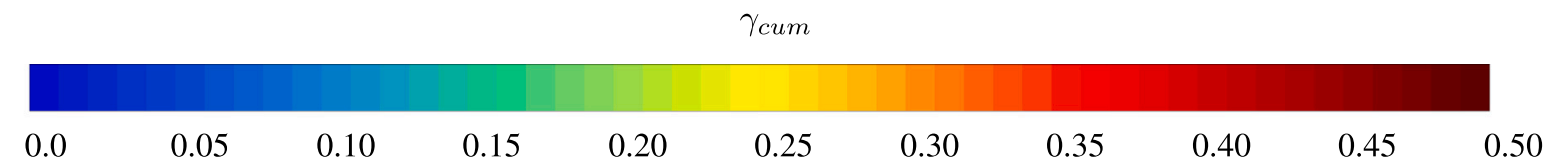

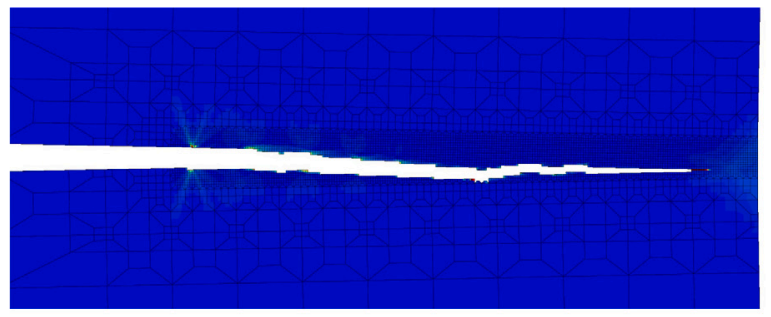

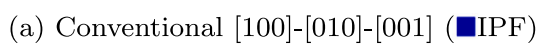

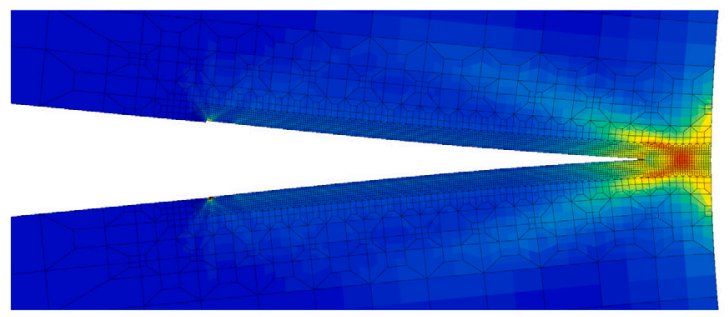

(c) Conventional [011]-[100]-[01̄̄] (\$IPF)

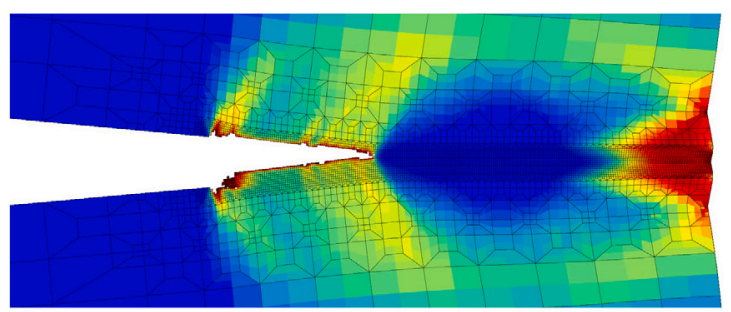

(e) Conventional [110]-[111]-[112] $(\varangle \mathrm{IPF})$

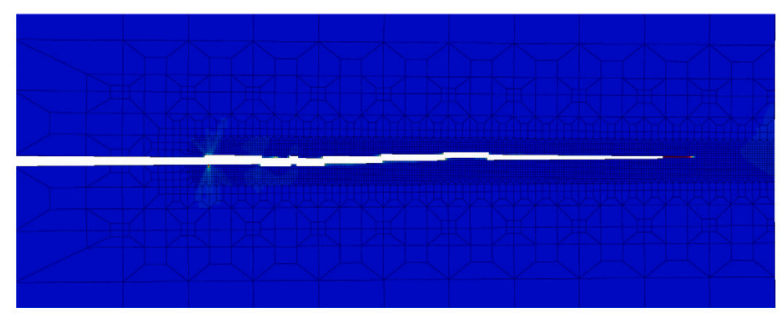

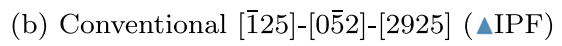

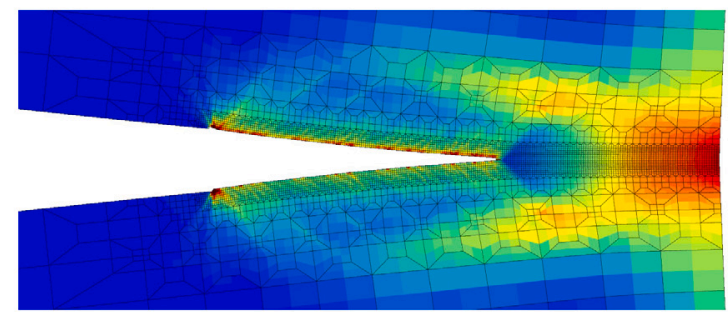

(d) Conventional [100]-[011]-[0̄11] (

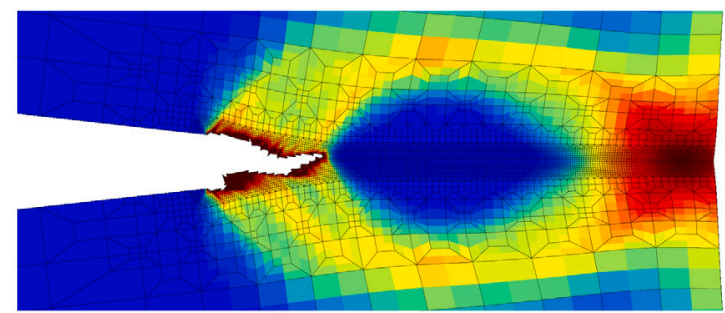

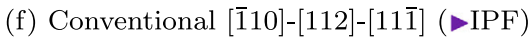

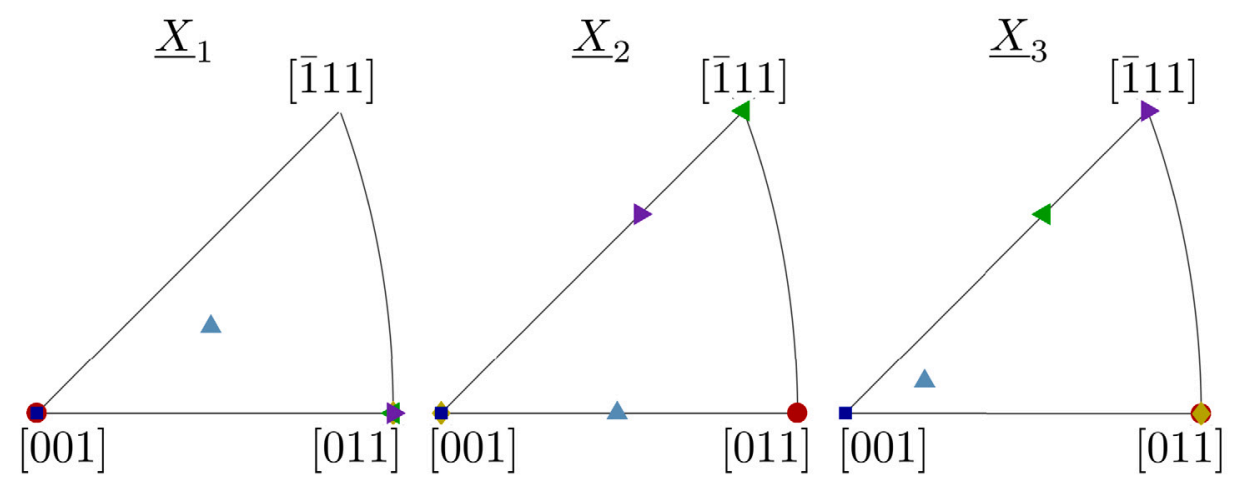

(g) inverse pole figure for the single crystal specimens

Fig. 9. Accumulated plastic slip fields in the vicinity of single crystal CT specimen notch front for several initial crystal orientations obtained with the conventional porous crystal plasticity model. The last computed time steps are shown. 
$\gamma_{\text {cum }}$

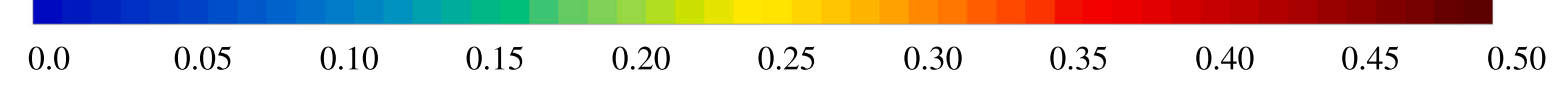

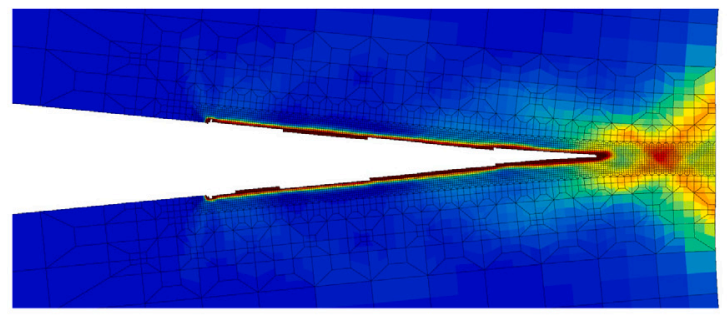

(a) Strain gradient [100]-[010]-[001] (泪F)

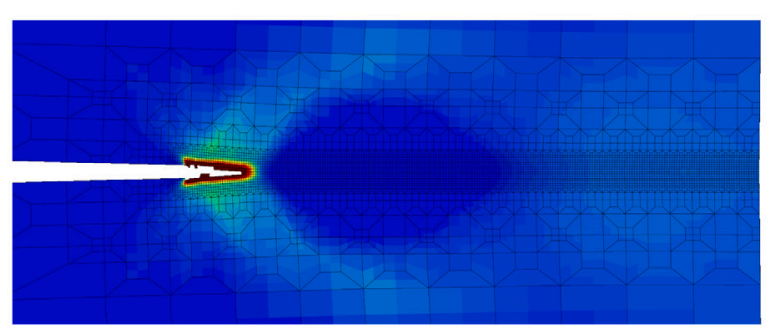

(c) Strain gradient [011]-[100]-[011]] $(\diamond \mathrm{IPF})$

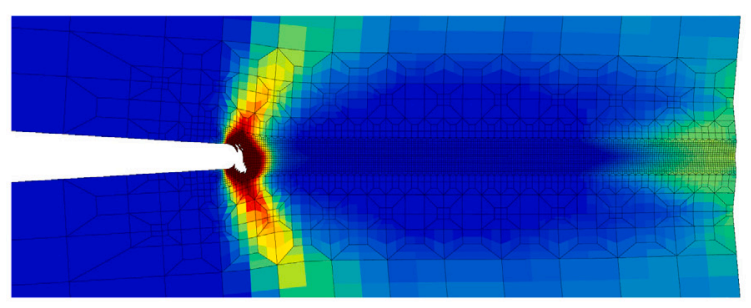

(e) Strain gradient [1]10]-[111]-[112] $(\triangleleft \mathrm{IPF})$

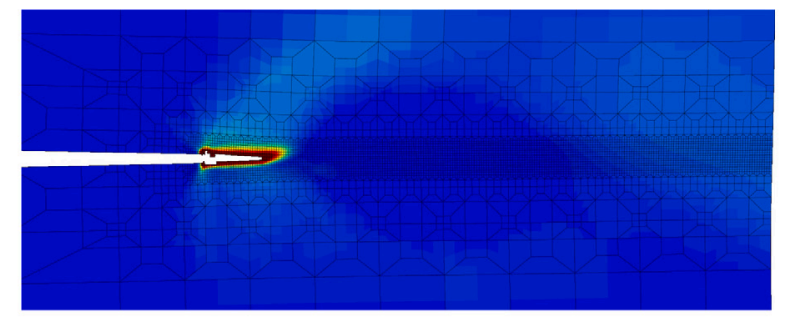

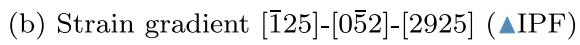

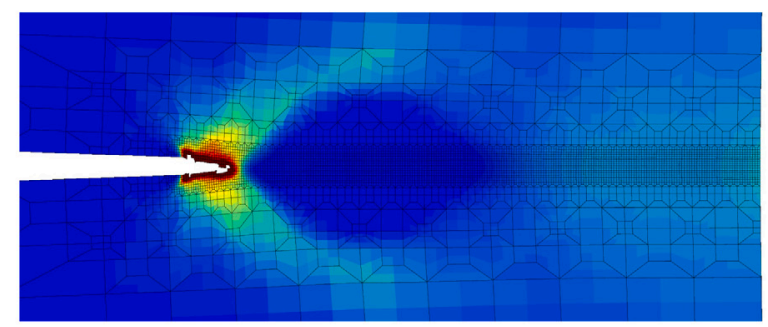

(d) Strain gradient [100]-[011]-[01̄1] $(\bullet \mathrm{IPF})$

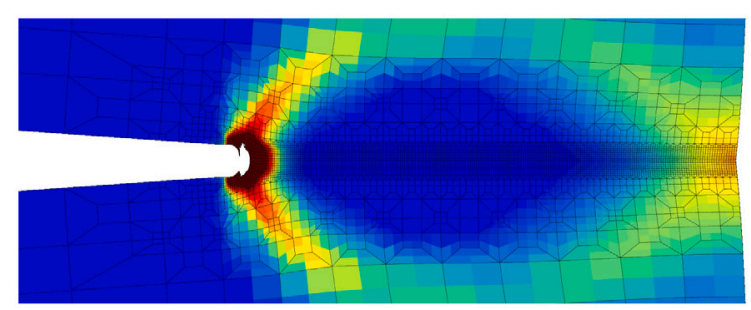

(f) Strain gradient [1110]-[112]-[111̄] $(\bullet \mathrm{IPF})$

Fig. 10. Accumulated plastic slip fields in the vicinity of single crystal CT specimen notch front for several initial crystal orientations obtained with the strain gradient porous crystal plasticity model $(A=1 \mathrm{~N})$. The last computed time steps are shown.

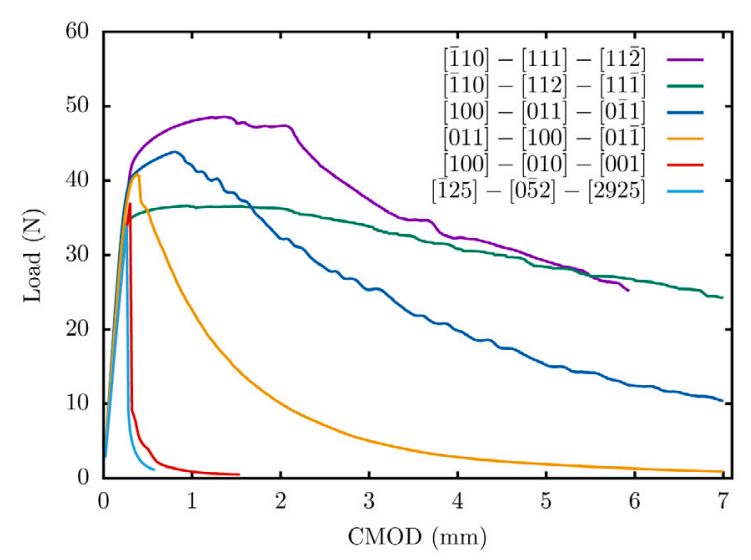

(a) Conventional

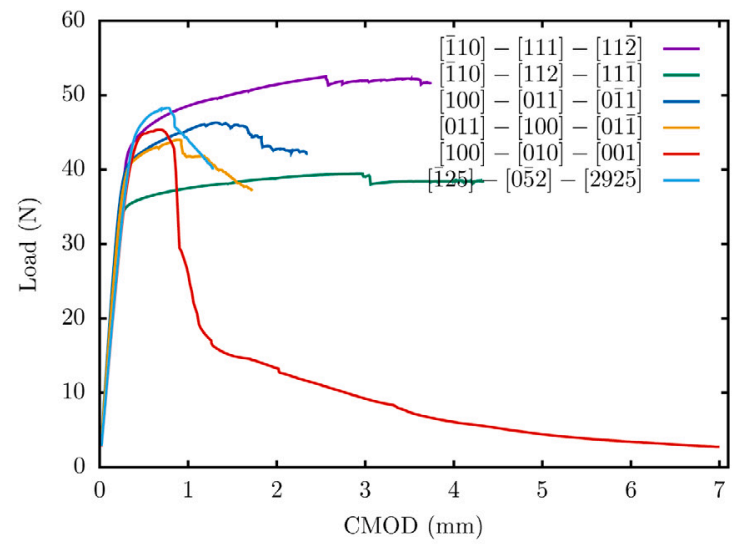

(b) Strain gradient $(A=1 \mathrm{~N})$

Fig. 11. Load $v s$ CMOD for conventional (a) and strain gradient (b) porous single crystal CT specimens. 


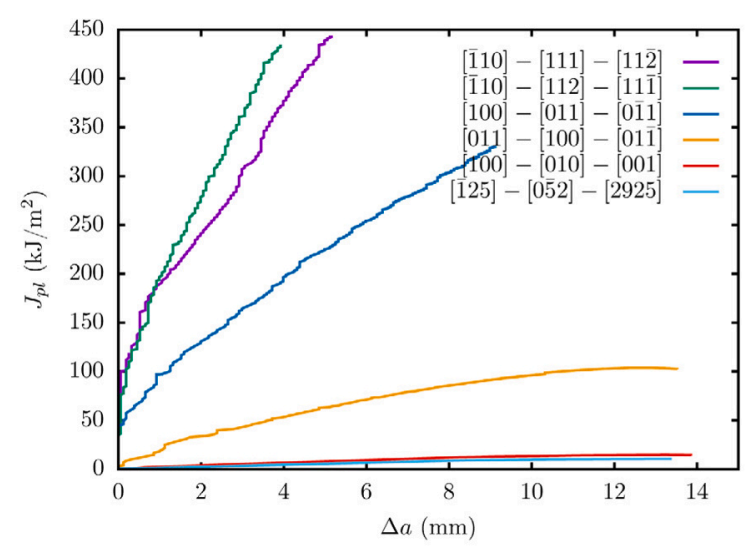

(a) Conventional

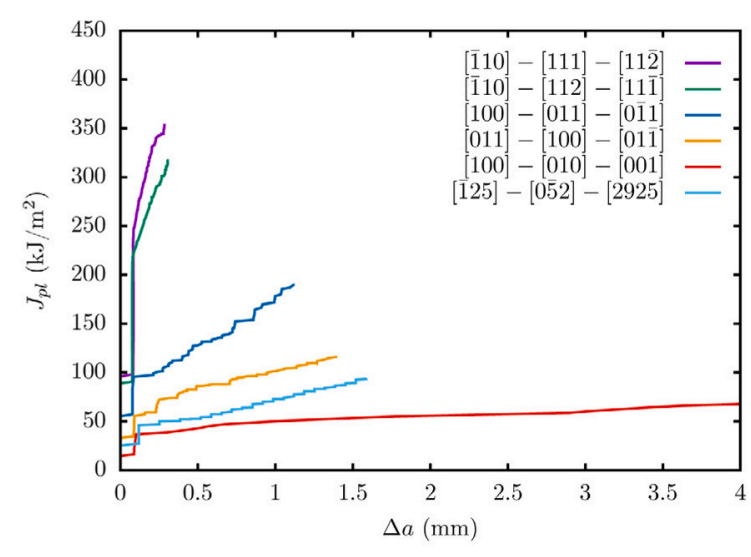

(b) Strain gradient $(A=1 \mathrm{~N})$

Fig. 12. $J_{p l}$ plastic component of $J v s$ crack extension $\Delta a$ for conventional (a) and strain gradient (b) porous single crystal CT specimens.

$\begin{array}{lllllll}0.20 & 0.25 & 0.30 & 0.35 & 0.40 & 0.45 & 0.50\end{array}$

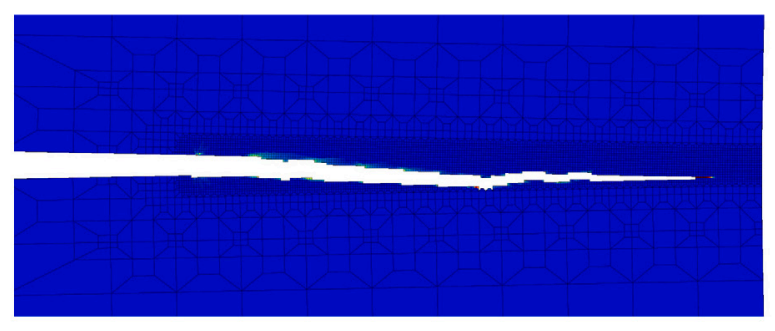

(a) Conventional

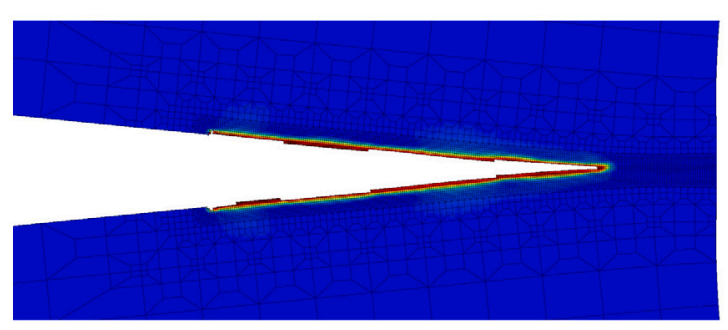

(c) Strain gradient $(A=1 \mathrm{~N})$

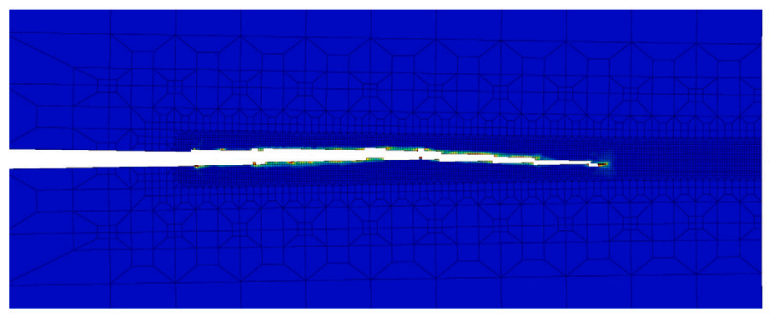

(b) Strain gradient $(A=0.1 \mathrm{~N})$

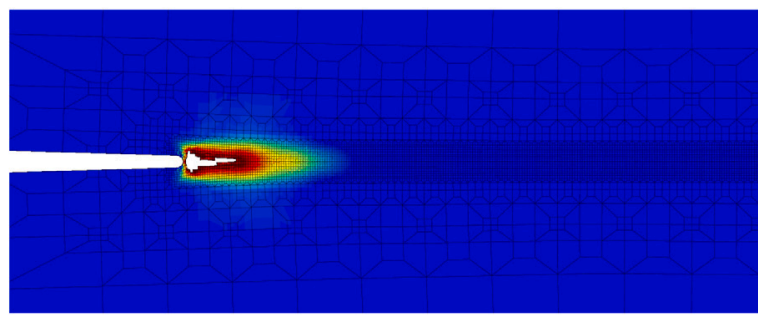

(d) Strain gradient $(A=10 \mathrm{~N})$

Fig. 13. Fields of damage variable $\chi$ (see Eq. (33)) for conventional and strain gradient porous [100]-[010]-[001] single crystal CT specimens with an initial porosity of $1 \%$. The last computed time step is shown.

with an initial porosity of $1 \%$. Nevertheless, a higher load is reached before onset of crack propagation with $f_{0}=0.1 \%$. The value of $J_{p l}$ reached at onset is hence much larger for an initial porosity of $0.1 \%$ than for an initial porosity of $1 \%$. These results echo the study of Pardoen and Hutchinson (2003) who have shown that the fracture toughness is a decreasing function of the initial void volume fraction and an increasing function of the intervoid distance. In the present work, the strain gradient modulus $A$ controls the internal length of the material. Therefore, it can be put in parallel to the characteristic intervoid distance $X_{0}$ used by Pardoen and Hutchinson (2003).

To study the propagation of cracks at the single crystal scale is a difficult task for materials such as austenitic stainless steels with conventional grain sizes of a few tenth of microns. The reason for that is that the standardized specimens sizes are usually much larger than the grain size. For such polycrystal specimens, the crystal orientation can be measured on the surface but can 


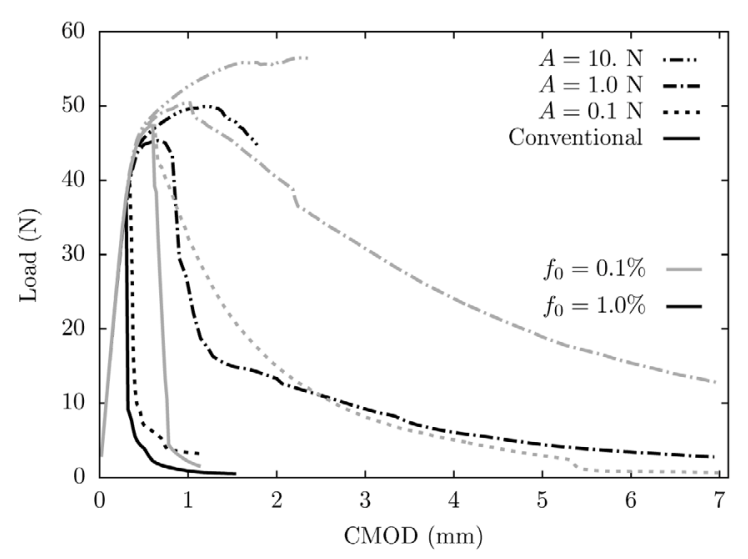

(a)

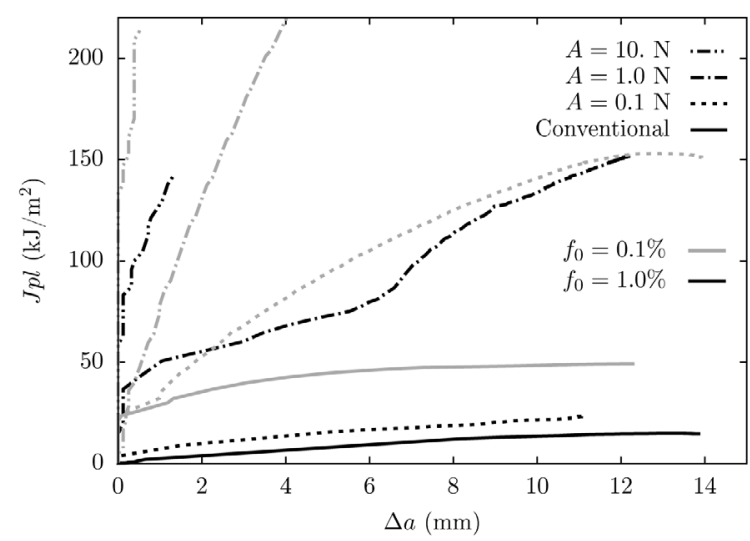

(b)

Fig. 14. Load $v s$ CMOD (a) and $J_{p l} v s$ crack extension $\Delta a$ (b) for conventional and strain gradient porous single crystal [100]-[010]-[001] CT specimen with initial porosity $f_{0}$ of $0.1 \%$ and $1.0 \%$.

hardly be obtained in the bulk of the specimen with non-destructive methods that would allow for subsequent mechanical testing. In addition the fact that such specimens are composed of numerous grains and grain boundaries makes it difficult to interpret the role of the crystal anisotropy on ductility and toughness. These shortcomings can be overcome for instance by designing model experiments. Small scale experiments can be considered in order to focus the observation on a few number of grains. However such kind of investigations do not come without additional complexity, since they involve the use of small scale experimental techniques and size effects can arise when the specimen size gets closer to the characteristic size of the deformation mechanisms. Alternatively, model materials which can naturally grow large grains (e.g. aluminum alloys) can be used to produce specimens composed of a few number of grains. These specimens can in turn be used to unravel the role of the crystal anisotropy in the ductile failure process. Studies of this kind for ductile materials are very scarce in the literature and are therefore work in progress by the authors in order to confront their numerical results to experimental data. Some studies have investigated experimentally the crack-tip stress-strain field in ductile materials (Shield and Kim, 1994; Kysar and Briant, 2002; Marchal et al., 2006) and validated the associated theory of stress sectors developed by Rice (1987). Following on from this work, current efforts are thus devoted to the characterization of the role of crystal plasticity on void growth and coalescence at the crack tip in order to assess the influence of crystal orientation on fracture toughness.

\subsection{Oligo-crystal CT specimen}

In order to investigate the interaction between the crack front and grain boundaries, a CT specimen composed of an artificial grain microstructure is studied. The specimen is divided in six rectangular grains along the $\underline{X}_{1}$ direction. Grain boundaries are therefore orthogonal to the direction of crack propagation. Fig. 15 shows the propagation of a crack in this ideal oligo-crystal specimen with the conventional and the strain gradient plasticity models. Each color shown in Figs. 15(a) and 15(c) indicates a different initial crystal orientation, while Figs. 15(b) and 15(d) display the accumulated plastic slip contour in the polycrystal specimen. Initial crystal orientations of the six different crystals where selected randomly and are presented in the form of Euler angles in Table 4 following the Bunge convention (ZXZ). The inverse pole figures of the six grains are shown in Fig. 15(e). With the conventional model, the crack propagates first out of the middle plane when advancing in grain \#2. Then, after reaching the grain boundary between grains \#2 and \#3, the crack moves straight along a horizontal plane until it reaches a second grain boundary. Ultimately, the crack bifurcates again when it starts propagating in grain \#4. In grains \#2 and \#4, in which the crack path is slanted with respect to the specimen horizontal symmetry plane, large levels of accumulated plastic slip are reached and cover wide areas above and below the crack lips. On the contrary, in grain \#3, in which the crack propagates horizontally, much less plastic activity is present in the vicinity of the crack lips. This observation demonstrates again that all crystal orientations are not equivalent in terms of crack propagation. The crack path predicted with the strain gradient plasticity model is also slanted in grain \#2, although the angle with respect to the horizontal axis is smaller than with the conventional model. The plastic activity covers an even wider area below and above the crack lips as compared to the conventional model. Interestingly, a pronounced shielding of plastic activity seems to occur at the grain boundary between grains \#2 and \#3. In addition, the plastic field is much more non-symmetric with respect to the horizontal symmetry plane than the plastic field predicted by the conventional model.

The load $v s$ CMOD and $J_{p l} v s \Delta a$ graphs shown in Fig. 16 display three very distinct regimes for the conventional model. A different color is used in these plots in order to indicate the grain in which the crack front is located at the current time step. After the elastic phase, the two grains displaying substantial plastic activity are characterized by regimes during which the load increases or decreases slowly with the CMOD. During these two regimes the crack propagates at a slow pace and the $J_{p l} v s \Delta a$ curves are thus steep. On the other hand, in between grain \#2 and grain \#4 the crack propagates at a fast rate in grain \#3. The load drops 


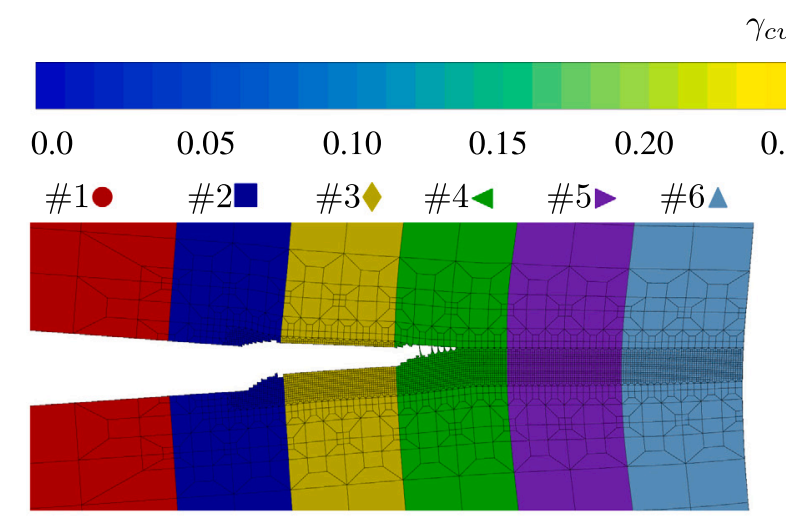

(a) Conventional polycrystal

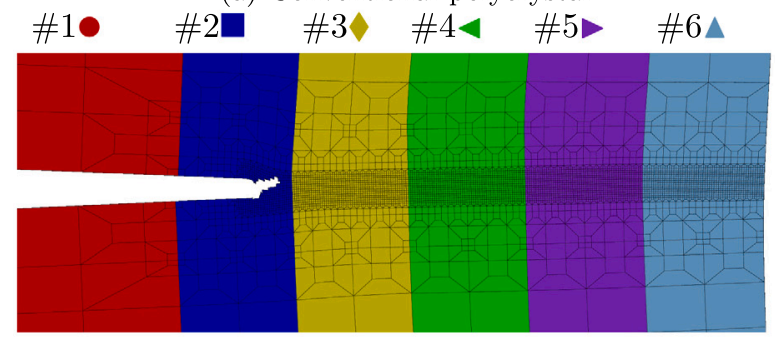

(c) Strain gradient polycrystal

$\gamma_{\text {cum }}$

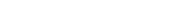

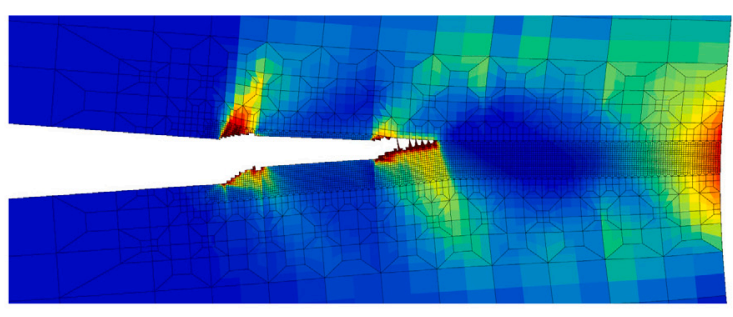

(b) Conventional polycrystal

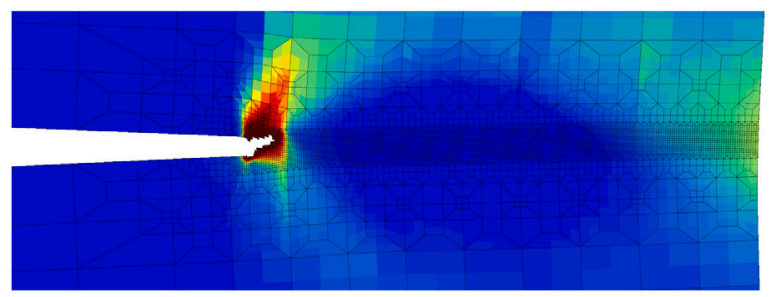

(d) Strain gradient polycrystal

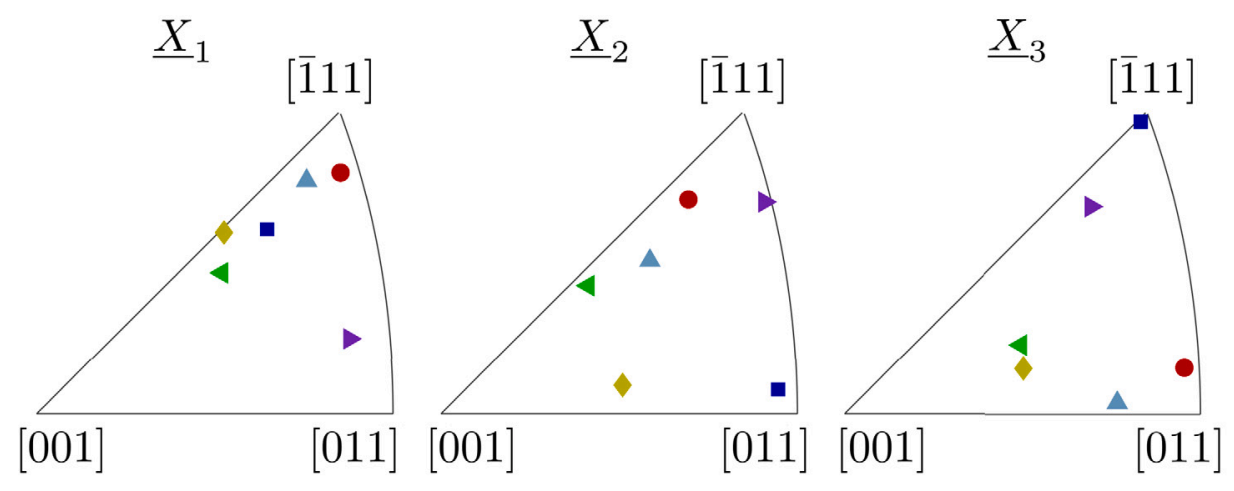

(e) inverse pole figure for the polycrystal specimen

Fig. 15. Accumulated plastic slip fields in the vicinity of a polycrystal CT specimen notch front obtained with the conventional and strain gradient porous crystal plasticity model. The last computed time step is shown. In Figures (a) and (c) each color represents a different initial crystal orientation reported in the inverse pole figure (e).

therefore rapidly and the plastic component of $J$ increases slowly. The strain gradient plasticity model predicts a tougher mechanical behaviour of the polycrystal. Since the crack displays more blunting than with the conventional model, the crack extension $\Delta a$ is smaller. The applied load and measured values of $J_{p l}$ are consequently larger with the strain gradient plasticity model.

The microstructure used here is obviously very simplistic and is not meant to be representative of any real material. Nevertheless, such an idealized situation allows to highlight more precisely the paramount role of the crystal plasticity anisotropy on the crack propagation and orientation-dependent ductility. Although it is not the objective of the present work, such oligo-crystal microstructure could, to some extent, be relevant for materials obtained by directional solidification such as for example columnar nickel-based superalloys (Giamei, 2013; Coudon et al., 2019). More importantly, with the advent of recent manufacturing processes (for instance additive manufacturing) design of complex microstructures are now within reach. The model and simulations presented in this work are a step forward towards designing and optimizing microstructures in order to fulfil engineering needs. Future work will be devoted to the simulation of real microstructures and to the comparison of numerical predictions with experimental data. 
Table 4

Euler angles $\left(\phi_{1}, \Phi, \phi_{2}\right)$ in degrees defining the initial crystal orientation of the six grains of the polycrystal CT specimen.

\begin{tabular}{llll}
\hline Grains & $\phi_{1}$ & $\Phi$ & $\phi_{2}$ \\
\hline \#1・ & 3.48 & 53.17 & 315.41 \\
\#2 & 310.96 & 84.69 & 136.94 \\
\#3 & 335.60 & 112.22 & 83.99 \\
\#4 & 76.48 & 40.56 & 139.94 \\
\#5 & 19.49 & 111.84 & 171.00 \\
\#6 & 34.35 & 34.17 & 267.59 \\
\hline
\end{tabular}

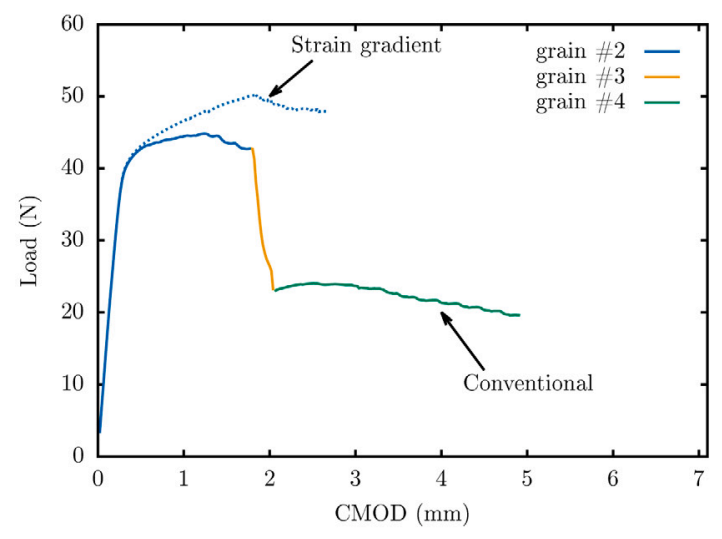

(a)

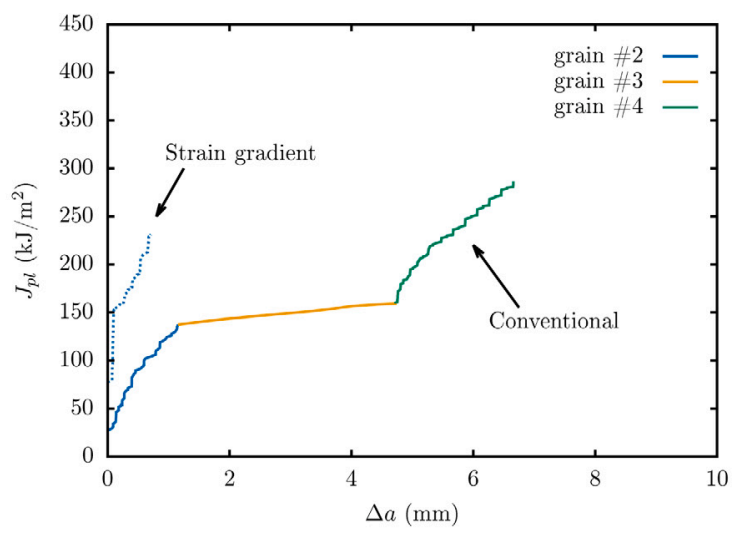

(b)

Fig. 16. Load $v s$ CMOD (a) and $J_{p l} v s$ crack extension $\Delta a$ (b) for porous oligo-crystal CT specimens.

\section{4. $3 D$ single crystal specimen}

Finally, a 3D [100]-[010]-[001] single crystal CT specimen was simulated. In this example, the plane strain boundary condition $\left(U_{3}=0\right)$ was relaxed. The specimen thickness is equal to $12.5 \mathrm{~mm}$. The 3D CT specimen mesh, shown in Fig. 17(a), is refined in the area of interest in order to have 18 elements across the thickness. The full 3D mesh contains 79980 quadratic elements composed each of 8 Gauss points and totals 1017249 DOF. An initial porosity of $1 \%$ and the same hardening material parameters as in previous section were used. Figs. 17 shows the finite element mesh, loading curves for the conventional and strain gradient plasticity models and several mechanical fields in the horizontal symmetry plane of the specimen for the conventional model. Since the stress triaxiality is greater inside the bulk of the specimen than on its surface, a curved crack front is observed as expected. The undamaged region is separated from the broken region by a layer of few elements undergoing void coalescence. In the broken region the stress vanishes. Therefore the crack front can also be noticed on the equivalent and mean stress contour plots. Interestingly the crack path is completely straight in this 3D specimen, while it was slanted with respect to the horizontal axis for the same crystal orientation in the 2D-plane strain simulation shown in 9(a). The large number of elements used in these simulations suggests that simulations of reasonably large and densely discretized microstructures are within reach with affordable computation times.

\section{Conclusions}

The main achievements and conclusions of this work can be listed as follows:

- A strain gradient porous crystal plasticity model was developed in a thermodynamically consistent framework at finite strains. The strain gradient approach relies on a Lagrange multiplier based extension of the free energy potential in order to account for gradients of an accumulated plastic slip scalar field.

- A new criterion for void coalescence onset in single crystals was proposed and validated by means of porous unit-cell simulations. The criterion relies on a revisited version of Thomason's criterion, in which the effective coalescence flow stress is implicitly defined by the stress satisfying a GTN criterion.

- An alternative formulation to model void coalescence involving an effective porosity $f^{*}$ was compared to the plastic mechanism based void coalescence model. Both approaches were tested on single Gauss point simulations at fixed triaxiality and up to failure. An extension of the plastic mechanism based model was shown to enable control of the softening rate in the post-coalescence regime. 


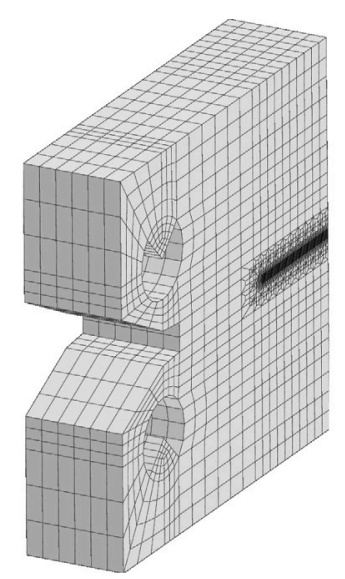

(a)

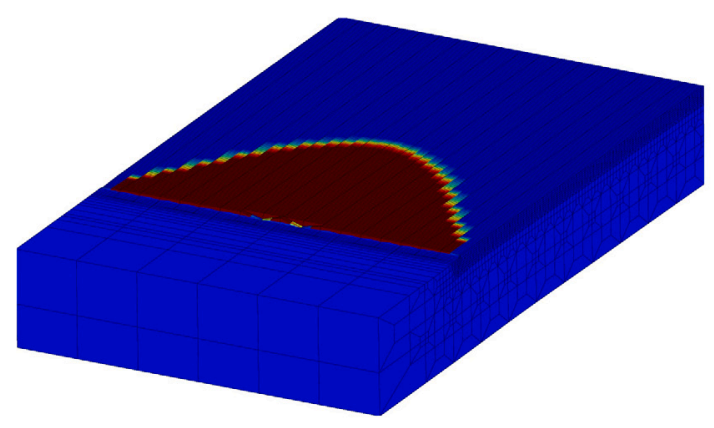
0.01 (c) $f^{*}$

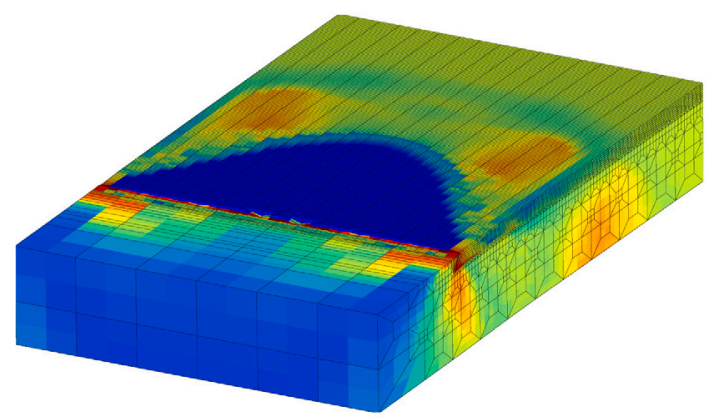

0
CMOD (mm)

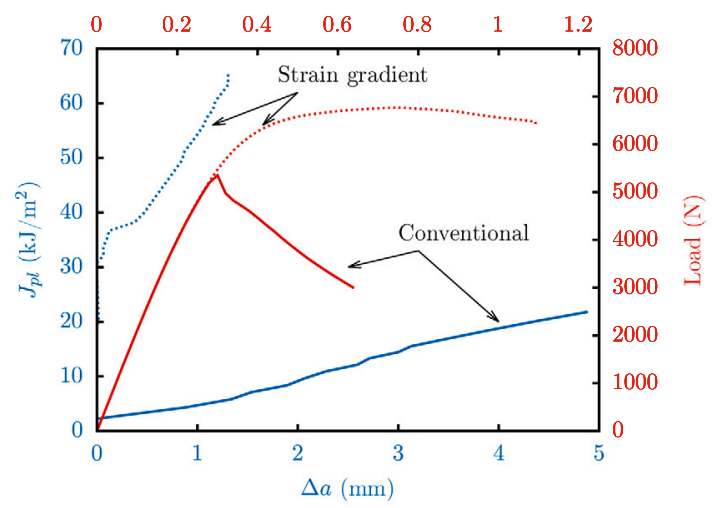

(b)

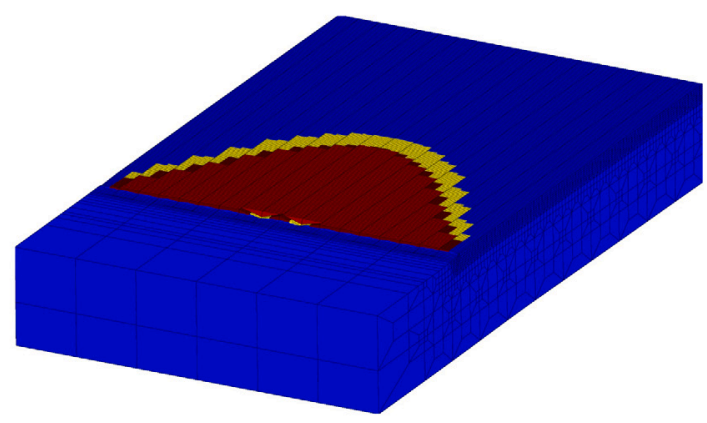

sound coalescence broken

(d) status

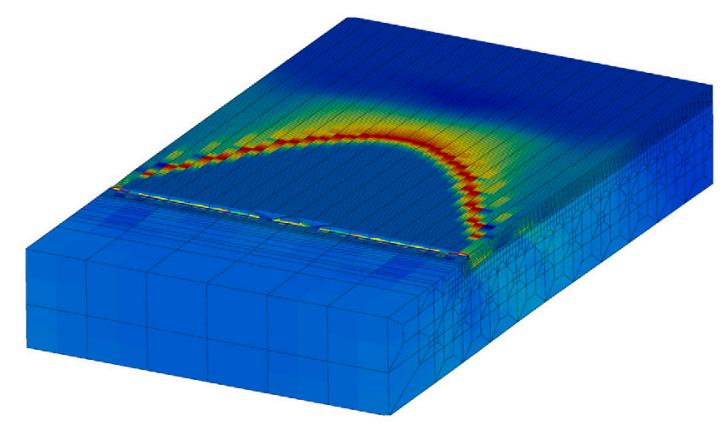

$-166$
900

(e) $\sigma_{e q}$

500

Fig. 17. Finite element mesh composed of 79980 quadratic elements (1017249 degrees of freedom) (a), $J_{p l} v s \Delta a$ for conventional and strain gradient models (b) and contour plots in the crack plane (c-f) for a three dimensional single crystal CT specimen with the conventional model. The crystal directions [100], [010] and [001] initially coincide with the orthonormal basis vectors $\underline{X}_{1}, \underline{X}_{2}$ and $\underline{X}_{3}$ respectively.

- The convergence with respect to mesh size when gradient terms are accounted for was demonstrated in simulations of a plate under plane strain tensile loading conditions. For sufficiently dense meshes the macroscopic stress $v s$ strain curves and local damage fields become indeed mesh size insensitive.

- First of a kind simulations of ductile fracture in porous single- and oligo-crystal plane strain CT-like geometries were performed.

The strain gradient plasticity model allows to regularize the width of the damaged area at the periphery of the crack. 
- A strongly anisotropic orientation-dependent failure behaviour is highlighted. Ductility and fracture toughness display a strong dependence on crystal orientation. Toughest orientations display the highest level of plastic activity in the vicinity of the crack lips and front.

- Crack bifurcation is observed with the conventional model for symmetric and non-symmetric crystal orientations. Decreasing the initial porosity or increasing the internal length scale in the strain gradient plasticity model leads to straight cracks for all orientations.

- Large scale simulations of a 3D single crystal CT specimen suggest that simulations of polycrystal microstructures are within reach with a satisfying resolution of intragranular mechanical fields.

The model and first simulations presented in this work open the way to the regularized simulation of ductile fracture in polycrystal microstructures. The framework which was developed can inherently account for the anisotropy due to crystal plasticity. Anisotropy due to morphological or crystallographic texture could also be incorporated without additional difficulties. The prospects of this work include the acquisition of experimental data at the scale of the microstructure. Microscale digital image correlation or in situ tomography tests would be appropriate techniques to obtain data to which numerical results could be confronted to. Moreover, numerical performance of implementation of this sort of models could still be enhanced. Special attention should be given to the treatment of crystal plasticity constitutive equations, to the conditioning of the differential system in the context of strain gradient plasticity and to the handling of fully damaged elements within the framework of fracture. These issues are possible tracks to further explore in order to enhance the computational efficiency of this numerical tool.

\section{CRediT authorship contribution statement}

Jean-Michel Scherer: Conceptualization, Methodology, Writing - original draft, Writing - review \& editing, Investigation, Software, Data curation. Jacques Besson: Conceptualization, Methodology, Writing - review \& editing, Software, Resources. Samuel Forest: Conceptualization, Methodology, Writing - review and Editing, Software, Resources. Jérémy Hure: Conceptualization, Methodology, Writing - review \& editing, Funding acquisition. Benoît Tanguy: Conceptualization, Methodology, Writing - review \& editing, Funding acquisition.

\section{Declaration of competing interest}

The authors declare that they have no known competing financial interests or personal relationships that could have appeared to influence the work reported in this paper.

\section{Acknowledgement}

The authors would like to thank G. Rousselier for valuable comments and fruitful discussions.

\section{Appendix. Single crystal porous unit-cell boundary conditions}

Periodic porous unit-cell finite element simulations are performed by prescribing a macroscopic deformation gradient $\bar{\sim}$ 的 to cubic cell containing an initially centered spherical void such that initial porosity $f_{0}=1 \%$. Periodic displacement boundary conditions are applied

$$
\left.\left.\underline{u}=\overline{\bar{F}} \cdot \underline{x}+\underline{v} \quad \underline{v}\left(\underline{x}^{+}\right)=\underline{v} \underline{x}^{-}\right) \quad \underline{T}\left(\underline{x}^{+}\right)=-\underline{T} \underline{x}^{-}\right)
$$

where $\underline{u}$ is the displacement field and $\underline{v}$ the periodic fluctuation. The vectors $\underline{x}^{+}$and $\underline{x}^{-}$denote homologous nodes on opposite faces of the unit-cell. In keeping with Ling et al. (2016) the macroscopic deformation gradient $\underset{\sim}{\overline{\boldsymbol{F}}}$ and first Piola-Kirchhoff stress $\underset{\sim}{\boldsymbol{S}}$ are related to their microscopic counterpart by volume averages

$$
\bar{\sim}=\frac{1}{V_{0}^{\text {tot }}} \int_{D_{0}}^{\underset{\sim}{\boldsymbol{F}} \mathrm{d} V \quad \bar{\sim}}=\frac{1}{V_{0}^{\text {tot }}} \int_{D_{0}} \underset{\sim}{\boldsymbol{S}} \mathrm{d} V
$$

where $V_{0}^{\text {tot }}$ denotes the total volume (including the void) of the unit-cell domain $D_{0}$ in the reference configuration. It follows that the macroscopic Cauchy stress is given by

$$
\bar{\sim}=\frac{1}{V^{t o t}} \int_{D} \underset{\sim}{\sigma} \mathrm{d} V=\frac{1}{\operatorname{det}(\underset{\sim}{\overline{\boldsymbol{F}}})} \bar{\sim} \underset{\sim}{\overline{\boldsymbol{S}}} \cdot \bar{\sim}^{T}
$$

where $V^{\text {tot }}$ denotes the total volume (void included, with suitable extension of the fields within the voids) of the unit-cell domain $D$ in the current configuration. Macroscopic hydrostatic $\left(\bar{\sigma}_{m}\right)$, equivalent $\left(\bar{\sigma}_{e q}\right)$ stresses are defined by

$$
\bar{\sigma}_{m}=\frac{\operatorname{tr}(\underset{\sim}{\bar{\sigma}})}{3} \quad \bar{\sigma}_{e q}=\sqrt{\frac{3}{2} \bar{\sim}^{\prime}: \bar{\sim}^{\prime}} \quad \bar{\sim}^{\prime}=\underset{\sim}{\bar{\sigma}}-\sigma_{m} \underset{\sim}{\mathbf{1}}
$$


Only axisymmetric loading conditions are considered for which the macroscopic stress tensor and stress triaxiality ratio can be written as

$$
\bar{\sim}=\left(\begin{array}{ccc}
\bar{\sigma}_{11} & 0 & 0 \\
0 & \eta \bar{\sigma}_{11} & 0 \\
0 & 0 & \eta \bar{\sigma}_{11}
\end{array}\right) \quad T=\frac{\bar{\sigma}_{m}}{\bar{\sigma}_{e q}}=\frac{1+2 \eta}{3(1-\eta)}
$$

The simulations are performed at fixed macroscopic Cauchy stress triaxialities $T \in\{1 ; 1.5 ; 2 ; 3\}$. The reader is referred to Ling et al. (2016) for the numerical implementation of such a condition.

\section{References}

Achouri, M., Germain, G., Dal Santo, P., Saidane, D., 2013. Experimental characterization and numerical modeling of micromechanical damage under different stress states. Mater. Des. 50, 207-222.

Aslan, O., Cordero, N.M., Gaubert, A., Forest, S., 2011. Micromorphic approach to single crystal plasticity and damage. Internat. J. Engrg. Sci. 49, 1311-1325. ASTM Standard E1820, 2017. Standard Test Method for Measurement of Fracture Toughness. American Society of Testing Materials, https://www.astm.org/.

Babout, L., Brechet, Y., Maire, E., Fougeres, R., 2004. On the competition between particle fracture and particle decohesion in metal matrix composites. Acta Mater. 52, 4517-4525.

Barrioz, P., Hure, J., Tanguy, B., 2019. Effect of dislocation channeling on void growth to coalescence in FCC crystals. Mater. Sci. Eng. A 749, 255-270.

Bažant, Z.P., Belytschko, T.B., Chang, T.-P., 1984. Continuum theory for strain-softening. J. Eng. Mech. 110, $1666-1692$.

Benzerga, A.A., Besson, J., 2001. Plastic potentials for anisotropic porous solids. Eur. J. Mech. A Solids 20, $397-434$.

Benzerga, A.A., Besson, J., Pineau, A., 1999. Coalescence-controlled anisotropic ductile fracture. Trans. ASME, J. Eng. Mater. Technol. 121 , 221-229.

Benzerga, A.A., Leblond, J.-B., 2010. Ductile fracture by void growth to coalescence. In: Advances in Applied Mechanics, volume 44. Elsevier, pp. 169-305.

Benzerga, A., Leblond, J.-B., 2014. Effective yield criterion accounting for microvoid coalescence. J. Appl. Mech. 81.

Bertram, A., 2012. Elasticity and Plasticity of Large Deformations. Springer.

Bertram, A., Glüge, R., 2016. Gradient materials with internal constraints. Math. Mech. Complex Syst. 4, 1-15.

Besson, J., 2009. Damage of ductile materials deforming under multiple plastic or viscoplastic mechanisms. Int. J. Plast. 25, $2204-2221$.

Besson, J., 2010. Continuum models of ductile fracture: A review. Int. J. Damage Mech. 19, 3-52.

Besson, J., Foerch, R., 1998. Object-oriented programming applied to the finite element method part I. General concepts. Rev. Eur. Eléments Finis 7, 535-566.

Besson, J., Guillemer-Neel, C., 2003. An extension of the Green and Gurson models to kinematic hardening. Mech. Mater. 35, 1-18.

Bouby, C., Kondo, D., 2017. Sur le cadre thermodynamique d'une classe de modèles de plasticité de milieux poreux ductiles. In: Congrès Français de Mécanique. AFM, Association Française de Mécanique.

Brepols, T., Wulfinghoff, S., Reese, S., 2017. Gradient-extended two-surface damage-plasticity: Micromorphic formulation and numerical aspects. Int. J. Plast. 97, 64-106.

Cao, T.-S., Mazière, M., Danas, K., Besson, J., 2015. A model for ductile damage prediction at low stress triaxialities incorporating void shape change and void rotation. Int. J. Solids Struct. 63, 240-263.

Chaboche, J.L., 1988. Continuum damage mechanics: Part I-General concepts. J. Appl. Mech. 55, 59-64.

Coudon, F., Cailletaud, G., Cormier, J., Marcin, L., 2019. A multiscale model for nickel-based directionally solidified materials. Int. J. Plast. 115 , 1-17.

Coussy, O., 2004. Poromechanics. John Wiley \& Sons.

Crépin, J., Bretheau, T., Caldemaison, D., 1996. Cavity growth and rupture of $\beta$-treated zirconium: A crystallographic model. Acta Mater. 44, $4927-4935$.

Danas, K., Castañeda, P.P., 2009. A finite-strain model for anisotropic viscoplastic porous media: I-Theory. Eur. J. Mech. A Solids 28, $387-401$.

Dormieux, L., Kondo, D., 2010. An extension of Gurson model incorporating interface stresses effects. Internat. J. Engrg. Sci. 48, 575-581.

Enakoutsa, K., Leblond, J.-B., 2009. Numerical implementation and assessment of the GLPD micromorphic model of ductile rupture. Eur. J. Mech. A Solids 28, $445-460$.

Enakoutsa, K., Leblond, J., Perrin, G., 2007. Numerical implementation and assessment of a phenomenological nonlocal model of ductile rupture. Comput. Methods Appl. Mech. Engrg. 196, 1946-1957.

Fleck, N., Hutchinson, J., 1997. Strain gradient plasticity. Adv. Appl. Mech. 33, 296-361.

Forest, S., Sievert, R., 2003. Elastoviscoplastic constitutive frameworks for generalized continua. Acta Mech. 160, 71-111.

Frodal, B.H., Thomesen, S., Børvik, T., Hopperstad, O.S., 2021. On the coupling of damage and single crystal plasticity for ductile polycrystalline materials. Int. J. Plast. 102996.

Gallican, V., Hure, J., 2017. Anisotropic coalescence criterion for nanoporous materials. J. Mech. Phys. Solids 108, 30-48.

Gambin, W., 1992. Refined analysis of elastic-plastic crystals. Int. J. Solids Struct. 29, 2013-2021.

Germain, P., Nguyen, Q.S., Suquet, P., 1983. Continuum thermodynamics. J. Appl. Mech. 50, 1010-1020.

Giamei, A.F., 2013. Development of single crystal superalloys: A brief history. Adv. Mater. Process. 171, 26-30.

Gologanu, M., Leblond, J.-B., Perrin, G., 1995. A micromechanically based Gurson-type model for ductile porous metals including strain gradient effects. In: Krishnaswami, S., McMeeking, R.M., Trasorras, J.R.I. (Eds.), In: Net Shape Processing of Powder Materials, vol. 216, ASME AMD, pp. 47-56.

Green, R., 1972. A plasticity theory for porous solids. Int. J. Mech. Sci. 14, 215-224.

Gurson, A.L., 1977. Continuum theory of ductile rupture by void nucleation and growth: Part I-Yield criteria and flow rules for porous ductile media. Trans. ASME, J. Eng. Mater. Technol. 99, 2-15.

Gurtin, M.E., Anand, L., 2009. Thermodynamics applied to gradient theories involving the accumulated plastic strain: The theories of Aifantis and Fleck and Hutchinson and their generalization. J. Mech. Phys. Solids 57, 405-421.

Ha, S., Kim, K., 2010. Void growth and coalescence in FCC single crystals. Int. J. Mech. Sci. 52, 863-873.

Håkansson, P., Wallin, M., Ristinmaa, M., 2006. Thermomechanical response of non-local porous material. Int. J. Plast. 22, 2066-2090.

Han, X., Besson, J., Forest, S., Tanguy, B., Bugat, S., 2013. A yield function for single crystals containing voids. Int. J. Solids Struct. 50, $2115-2131$.

Hill, R., 1948. A theory of the yielding and plastic flow of anisotropic metals. Proc. R. Soc. A 193, 281-297.

Holte, I., Niordson, C., Nielsen, K., Tvergaard, V., 2019. Investigation of a gradient enriched Gurson-Tvergaard model for porous strain hardening materials. Eur. J. Mech. A Solids 75, 472-484.

Hure, J., 2019. A coalescence criterion for porous single crystals. J. Mech. Phys. Solids 124, 505-525.

Hure, J., El Shawish, S., Cizelj, L., Tanguy, B., 2016. Intergranular stress distributions in polycrystalline aggregates of irradiated stainless steel. J. Nucl. Mater. 476, 231-242.

Kachanov, L., 2013. Introduction to Continuum Damage Mechanics. volume 10. Springer Science \& Business Media.

Keralavarma, S., Chockalingam, S., 2016. A criterion for void coalescence in anisotropic ductile materials. Int. J. Plast. 82, $159-176$.

Khadyko, M., Frodal, B.H., Hopperstad, O.S., 2021. Finite element simulation of ductile fracture in polycrystalline materials using a regularized porous crystal plasticity model. Int. J. Fract. 228, 15-31. 
Kubin, L., Devincre, B., Hoc, T., 2008. Modeling dislocation storage rates and mean free paths in face-centered cubic crystals. Acta Mater. 56, 6040-6049. Kysar, J.W., Briant, C.L., 2002. Crack tip deformation fields in ductile single crystals. Acta Mater. 50, $2367-2380$.

Lindroos, M., Laukkanen, A., Andersson, T., Vaara, J., Mäntylä, A., Frondelius, T., 2019. Micromechanical modeling of short crack nucleation and growth in high cycle fatigue of martensitic microstructures. Comput. Mater. Sci. 170, 109185.

Lindroos, M., Scherer, J.-M., Forest, S., Laukkanen, A., Andersson, T., Vaarac, J., Mäntylä, Antti, Frondelius, T., 2020. Micromorphic Crystal Plasticity Approach to Damageregularization and Size Effects in Martensitic Steels. In review.

Ling, C., Besson, J., Forest, S., Tanguy, B., Latourte, F., Bosso, E., 2016. An elastoviscoplastic model for porous single crystals at finite strains and its assessment based on unit cell simulations. Int. J. Plast. 84, 58-87.

Ling, C., Forest, S., Besson, J., Tanguy, B., Latourte, F., 2018. A reduced micromorphic single crystal plasticity model at finite deformations. Application to strain localization and void growth in ductile metals. Int. J. Solids Struct. 134, 43-69.

Lorentz, E., Benallal, A., 2005. Gradient constitutive relations: Numerical aspects and application to gradient damage. Comput. Methods Appl. Mech. Engrg. 194, 5191-5220.

Lorentz, E., Besson, J., Cano, V., 2008. Numerical simulation of ductile fracture with the Rousselier constitutive law. Comput. Methods Appl. Mech. Engrg. 197, 1965-1982.

Marchal, N., Flouriot, S., Forest, S., Rémy, L., 2006. Crack-tip stress-strain fields in single crystal nickel-base superalloys at high temperature under cyclic loading. Comput. Mater. Sci. 37, 42-50.

Mbiakop, A., Constantinescu, A., Danas, K., 2015. An analytical model for porous single crystals with ellipsoidal voids. J. Mech. Phys. Solids 84, $436-467$.

McClintock, F.A., 1968. A criterion for ductile fracture by the growth of holes. J. Appl. Mech. 35, 363-371.

Mear, M.E., Hutchinson, J., 1985. Influence of yield surface curvature on flow localization in dilatant plasticity. Mech. Mater. 4, $395-407$.

Miehe, C., Kienle, D., Aldakheel, F., Teichtmeister, S., 2016. Phase field modeling of fracture in porous plasticity: A variational gradient-extended Eulerian framework for the macroscopic analysis of ductile failure. Comput. Methods Appl. Mech. Engrg. 312, 3-50.

Monchiet, V., Kondo, D., 2013. Combined voids size and shape effects on the macroscopic criterion of ductile nanoporous materials. Int. J. Plast. 43, $20-41$.

Mori, T., Meshii, M., 1969. Plastic deformation of quench-hardened aluminum single crystals. Acta Metall. 17, 167-175.

Morin, L., Leblond, J.-B., Benzerga, A.A., 2015. Coalescence of voids by internal necking: Theoretical estimates and numerical results. J. Mech. Phys. Solids 75, 140-158.

Morin, L., Michel, J.-C., Leblond, J.-B., 2017. A Gurson-type layer model for ductile porous solids with isotropic and kinematic hardening. Int. J. Solids Struct. 118, 167-178.

Nahshon, K., Hutchinson, J., 2008. Modification of the Gurson model for shear failure. Eur. J. Mech. A Solids $27,1$.

Nguyen, V.-D., Lani, F., Pardoen, T., Morelle, X., Noels, L., 2016. A large strain hyperelastic viscoelastic-viscoplastic-damage constitutive model based on a multi-mechanism non-local damage continuum for amorphous glassy polymers. Int. J. Solids Struct. 96, 192-216.

Nguyen, V.-D., Pardoen, T., Noels, L., 2020. A nonlocal approach of ductile failure incorporating void growth, internal necking, and shear dominated coalescence mechanisms. J. Mech. Phys. Solids 137, 103891.

Niordson, C.F., Tvergaard, V., 2019. A homogenized model for size-effects in porous metals. J. Mech. Phys. Solids 123, $222-233$.

Noell, P.J., Carroll, J.D., Boyce, B.L., 2018. The mechanisms of ductile rupture. Acta Mater. 161, 83-98.

Nouailhas, D., Cailletaud, G., 1992. Comparaison de divers critères anisotropes pour monocristaux cubiques à face centrée (CFC). C. R. Acad. Sci. II 315, $1573-1579$.

Pardoen, T., Hutchinson, J., 2000. An extended model for void growth and coalescence. J. Mech. Phys. Solids 48, 2467-2512.

Pardoen, T., Hutchinson, J., 2003. Micromechanics-based model for trends in toughness of ductile metals. Acta Mater. 51, $133-148$.

Pascon, J.P., Waisman, H., 2020. A thermodynamic framework to predict ductile damage in thermoviscoplastic porous metals. Mech. Mater. 103701.

Paux, J., Brenner, R., Kondo, D., 2018. Plastic yield criterion and hardening of porous single crystals. Int. J. Solids Struct. 132 , 80-95.

Payet, S.F., Besson, J., Feyel, F., Chiaruttini, V., 2012. Crack initiation and propagation in nonlocal ductile media. In: ICDM. p. 117.

Pineau, A., Benzerga, A.A., Pardoen, T., 2016. Failure of metals I: Brittle and ductile fracture. Acta Mater. 107, 424-483.

Poh, L.H., Sun, G., 2017. Localizing gradient damage model with decreasing interactions. Internat. J. Numer. Methods Engrg. 110, 503-522.

Potirniche, G., Hearndon, J., Horstemeyer, M., Ling, X., 2006. Lattice orientation effects on void growth and coalescence in FCC single crystals. Int. J. Plast. 22, 921-942.

Ramaswamy, S., Aravas, N., 1998. Finite element implementation of gradient plasticity models Part II: Gradient-dependent evolution equations. Comput. Methods Appl. Mech. Engrg. 163, 33-53.

Reusch, F., Svendsen, B., Klingbeil, D., 2003. A non-local extension of Gurson-based ductile damage modeling. Comput. Mater. Sci. 26, $219-229$.

Rice, J.R., 1987. Tensile crack tip fields in elastic-ideally plastic crystals. Mech. Mater. 6, 317-335.

Rice, J.R., Tracey, D.M., 1969. On the ductile enlargement of voids in triaxial stress fields. J. Mech. Phys. Solids 17, $201-217$.

Rousselier, G., 1981. Finite deformation constitutive relations including ductile fracture damage. In: Nemat-Nasser, S. (Ed.), IUTAM Symposium. In: Three-Dimensional Constitutive Relations and Ductile Fracture, North-Holland, Amsterdam, pp. 319-343.

Rousselier, G., 1987. Ductile fracture models and their potential in local approach of fracture. Nucl. Eng. Des. 105, 97-111.

Rousselier, G., 2001. Dissipation in porous metal plasticity and ductile fracture. J. Mech. Phys. Solids 49, 1727-1746.

Scherer, J.-M., 2020. Strain Localization and Ductile Fracture in Single Crystals: Application to Irradiated Austenitic Stainless Steels (Ph.D. thesis). Paris Sciences et Lettres.

Scherer, J.-M., Besson, J., Forest, S., Hure, J., Tanguy, B., 2019. Strain gradient crystal plasticity with evolving length scale: Application to voided irradiated materials. Eur. J. Mech. A Solids 77, 103768.

Scherer, J.-M., Hure, J., 2019. A size-dependent ductile fracture model: Constitutive equations, numerical implementation and validation. Eur. J. Mech. A Solids 76, 135-145.

Scherer, J.-M., Phalke, V., Besson, J., Forest, S., Hure, J., Tanguy, B., 2020. Lagrange multiplier based vs micromorphic gradient-enhanced rate-(in) dependent crystal plasticity modelling and simulation. Comput. Methods Appl. Mech. Engrg. 372, 113426.

Scheyvaerts, F., Onck, P., Tekog̃lu, C., Pardoen, T., 2011. The growth and coalescence of ellipsoidal voids in plane strain under combined shear and tension. J. Mech. Phys. Solids 59, 373-397.

Selvarajou, B., Joshi, S.P., Benzerga, A.A., 2019. Void growth and coalescence in hexagonal close packed crystals. J. Mech. Phys. Solids 125 , $198-224$.

Shield, T., Kim, K.-S., 1994. Experimental measurement of the near tip strain field in an iron-silicon single crystal. J. Mech. Phys. Solids 42 , 845-873.

Song, D., Castañeda, P.P., 2017. A finite-strain homogenization model for viscoplastic porous single crystals: I-Theory. J. Mech. Phys. Solids 107, 560-579.

Thomason, P., 1985. Three-dimensional models for the plastic limit-loads at incipient failure of the intervoid matrix in ductile porous solids. Acta Metall. 33, 1079-1085.

Torki, M.E., Benzerga, A.A., 2018. A mechanism of failure in shear bands. Extreme Mech. Lett. 23, 67-71.

Torki, M., Tekoglu, C., Leblond, J.-B., Benzerga, A., 2017. Theoretical and numerical analysis of void coalescence in porous ductile solids under arbitrary loadings. Int. J. Plast. 91, 160-181.

Tvergaard, V., 1981. Influence of voids on shear band instabilities under plane strain conditions. Int. J. Fract. 17, $389-407$.

Tvergaard, V., 1982. On localization in ductile materials containing spherical voids. Int. J. Fract. 18, $237-252$.

Tvergaard, V., Needleman, A., 1984. Analysis of the cup-cone fracture in a round tensile bar. Acta Metall. 32, 157-169. 
Tvergaard, V., Needleman, A., 1995. Effects of nonlocal damage in porous plastic solids. Int. J. Solids Struct. 32, $1063-1077$.

Vishwakarma, V., Keralavarma, S.M., 2019. Micromechanical modeling and simulation of the loading path dependence of ductile failure by void growth to coalescence. Int. J. Solids Struct. 166, 135-153.

Wulfinghoff, S., Böhlke, T., 2012. Equivalent plastic strain gradient enhancement of single crystal plasticity: Theory and numerics. Proc. R. Soc. Lond. Ser. A Math. Phys. Eng. Sci. 468, 2682-2703.

Xue, Z., Pontin, M., Zok, F., Hutchinson, J.W., 2010. Calibration procedures for a computational model of ductile fracture. Eng. Fract. Mech. 77, 492-509.

Yerra, S., Tekog̃lu, C., Scheyvaerts, F., Delannay, L., Van Houtte, P., Pardoen, T., et al., 2010. Void growth and coalescence in single crystals. Int. J. Solids Struct. 47, 1016-1029.

Zhang, Y., Lorentz, E., Besson, J., 2018. Ductile damage modelling with locking-free regularised GTN model. Internat. J. Numer. Methods Engrg. 113, 1871-1903. Zhang, Z., Thaulow, C., Ødegård, J., 2000. A complete Gurson model approach for ductile fracture. Eng. Fract. Mech. 67, $155-168$.

Zybell, L., Hütter, G., Linse, T., Mühlich, U., Kuna, M., 2014. Size effects in ductile failure of porous materials containing two populations of voids. Eur. J. Mech. A Solids 45, 8-19. 